S. Niwa

Nagoya Math. J.

Vol. 108 (1987), 93-119

\title{
THE INNER PRODUCT OF AN AUTOMORPHIC WAVE FORM WITH THE PULLBACK OF AN EISENSTEIN SERIES
}

\author{
SHINJI NIWA
}

In this paper we shall show a relation between a special value of an automorphic wave form and the inner product of the automorphic wave form with the pullback of an Eisenstein series on the upper half space. The main theorem is Theorem 3 in the end of this paper. As is shown in P. B. Garrett [13], pullbacks of Eisenstein series on Siegel upper half spaces have interesting properties as a kernel function of an integral operator. It is natural to try to investigate pullbacks of Eisenstein series of Hilbert type. We can say that Theorem 3 clarifies a property of such pullbacks in a special case. The idea of the proof is a lifting of automorphic forms by theta functions. We discuss a lifting of automorphic wave forms in 1,2 and 3, and obtain Theorem 2 in the end of 3 as a result. We can prove Theorem 3 without much difficulty by using Theorem 2.

1. We denote, as usual, by $Z, Q, R$ and $C$ the ring of rational integers, the rational number field the real and the complex number fields. For $z \in C$, we define $\sqrt{z}=z^{1 / 2}$ so that $-\pi / 2<\arg \left(z^{1 / 2}\right) \leq \pi / 2$.

We discuss a lifting of an automorphic wave form by means of theta functions for a quadratic form with the signature $(+2,-2)$. Denote by $H$ the upper half plane. For $g_{1}, g_{2} \in G=G_{\infty}=S L(2, R)$, put

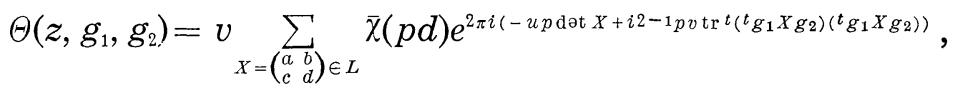

$$
\begin{aligned}
& \tilde{\Theta}\left(z, g_{1}, g_{2}\right)=v \sum_{X=\left(\begin{array}{cc}
a b \\
c & d
\end{array}\right) \in \tilde{L}} \chi(a) e^{2 \pi i\left(-u \operatorname{det} X+i 2-1 v \operatorname{tr} t\left(t g_{1} X g_{2}\right)\left(t g_{1} X g_{2}\right)\right)}
\end{aligned}
$$

where

$$
L=\left\{\left(\begin{array}{ll}
a & b \\
c & d
\end{array}\right) \mid a, b, c, p d \in Z\right\}, \tilde{L}=\left\{\left(\begin{array}{ll}
a & b \\
c & d
\end{array}\right) \mid a, b, c, d \in Z\right\}, \quad z=u+i v \in H
$$

Received June 24, 1986. 
and $\chi$ is a primitive character modulo a prime $p \equiv 1(\bmod 4)$. Assume $\chi(-1)=1$. We consider $\chi$ as a character on $Z_{p}$ by extending $\chi$ to a character on $Z_{p}^{\times}$in the usual way and putting $\chi(p)=0$. Then we have

Proposition 1.

$$
\Theta\left(-1 / p z, g_{1}, g_{2}\right)=p^{-1} g(\bar{\chi}) \tilde{\Theta}\left(z, g_{1}, g_{2}\right),
$$

with $g(\chi)=\sum_{l \bmod p} \chi(l) e^{2 \pi i l / p}$ and for $\left(\begin{array}{ll}a & b \\ c & d\end{array}\right) \in \Gamma=\Gamma_{0}(p)$,

$$
\begin{aligned}
& \Theta\left(\gamma z, g_{1}, g_{2}\right)=\bar{\chi}(d) \Theta\left(z, g_{1}, g_{2}\right), \\
& \Theta\left(z, \gamma g_{1}, g_{2}\right)=\Theta\left(z, g_{1}, \gamma g_{2}\right)=\chi(d) \Theta\left(z, g_{1}, g_{2}\right), \\
& \tilde{\Theta}\left(\gamma z, g_{1}, g_{2}\right)=\chi(d) \tilde{\Theta}\left(z, g_{1}, g_{2}\right), \\
& \tilde{\Theta}\left(z, \gamma g_{1}, g_{2}\right)=\tilde{\Theta}\left(z, g_{1}, \gamma g_{2}\right)=\chi(d) \tilde{\Theta}\left(z, g_{1}, g_{2}\right) .
\end{aligned}
$$

Proof. It is sufficient to prove (1.3), (1.4) and (1.5). Put $X=\left(\begin{array}{ll}m & n \\ x & y\end{array}\right)$ $\in L$ and $\gamma=\left(\begin{array}{ll}a & b \\ c & d\end{array}\right) \in \Gamma$, then ${ }^{t \gamma-1} X=\left(\begin{array}{l}* \\ *-b n+a y\end{array}\right) \in L$ and $\bar{\chi}(p(-b n+a y))$ $=\bar{\chi}(p a y)=\chi(d) \bar{\chi}(p y)$, which proves $\Theta\left(z, \gamma g_{1}, g_{2}\right)=\chi(d) \Theta\left(z, g_{1}, g_{2}\right)$. The proof of $\Theta\left(z, g_{1}, \gamma g_{2}\right)=\chi(d) \Theta\left(z, g_{1}, g_{2}\right)$ is the same. As for the proof of (1.3) and (1.4), we can apply transformation formulas in [7], which are summarized in [16] as Proposition 0, for example. However we describe the transformation formulas in the language of adeles to prove (1.3) and (1.4) for later use. For an archimedean or non-archimedean place $v, \boldsymbol{Q}_{v}$ denotes the completion at $v$. Let $A$ be the adele ring of $\boldsymbol{Q}$ and denote by $\varphi\left(K^{n}\right)$ the Schwartz Bruhat space on $K^{n}$ when $K=\boldsymbol{A}$ or $\boldsymbol{Q}_{v}$. Define an additive character $\psi_{v}$ (respectively $\psi$ ) of $\boldsymbol{Q}_{v}$ (respectively $\boldsymbol{A}$ ) by $\psi_{v}(x)=e^{2 \pi i \text { (the principal }}$ part of $x$ ) if $v$ is non-archimedean and $\psi_{v}(x)=e^{2 \pi i x}$ if $v$ is archimedean (respectively $\psi\left(\left(x_{v}\right)\right)=\prod_{v} \psi_{v}\left(x_{v}\right)$ ). Denote by $d_{v} x$ the Haar measure on $\boldsymbol{Q}_{v}$ normalized by $\int_{z_{q}} d_{q} x=1$ and $\int_{0}^{1} d_{\infty} x=1$. Put $d\left(x_{v}\right)=\prod_{v} d_{v} x$. Define a partial "Fourier" transformation $\mathscr{F}_{m, v}$ in $\mathscr{S}\left(\boldsymbol{Q}_{v}^{2}\right)$ by $\mathscr{F}_{m, v} f(x, y)=\int_{Q_{v}} f(x, z)$. $\psi_{v}(m z y) d_{v} z$ with $m \in \boldsymbol{Q}$ and a transformation $\lambda(g)$ in $\varphi\left(\boldsymbol{Q}_{v}^{2}\right)$ by $\lambda(g) f(x, y)$ $=f((x, y) g)$ for $g \in G_{v}=S L\left(2, \boldsymbol{Q}_{v}\right)$. Then $\mathscr{F}_{m, v}^{-1}=|m|_{v} \mathscr{F}_{-m, v}$. Define representations $r_{v, 1}, r_{v, 2}$ and $r_{v}$ of $G_{v}$ by $r_{v, 1}(g)=\mathscr{F}_{-p, v}^{-1} \lambda(g) \mathscr{F}_{-p, v}, r_{v, 2}(g)=$ $\mathscr{F}_{p, v}^{-1} \lambda(g) \mathscr{F}_{p, v}$ and $r_{v}(g)=r_{v, 1}(g) \otimes r_{r, 2}(g)$. Then the Poisson summation formula 


$$
\sum_{x \in Q^{2}}\left(\prod_{v} \mathscr{F}_{m, v}\right) f(x)=\sum_{x \in Q^{2}} f(x)
$$

is valid for $f \in \mathscr{S}\left(A^{2}\right)$, and therefore

$$
\sum_{x \in Q^{4}}\left(\prod_{v} r_{v}(g)\right) f(x)=\sum_{x \in Q^{4}} f(x)
$$

holds for $f \in \mathscr{S}\left(A^{4}\right)$ and $g \in G_{Q}=S L(2, Q)$. Define a representation $r$ of $G_{\boldsymbol{A}}=S L(2, \boldsymbol{A})$ on $\mathscr{S}\left(\boldsymbol{A}^{4}\right)$ by $r\left(\left(g_{v}\right)\right)=\prod_{v} r_{v}\left(g_{v}\right)$. When $K$ denotes $\boldsymbol{Q}_{v}$ or $\boldsymbol{A}$, we define a mapping $\alpha$ from $K^{4}$ to $M_{2,2}(K)$ by $\alpha(a, b, c, d)=\left(\begin{array}{ll}a & c \\ d & b\end{array}\right)$. Define a representation $\rho_{v}$ (respectively $\rho$ ) of $G_{v} \times G_{v}$ (respectively $G_{A} \times G_{A}$ ) on $\boldsymbol{Q}_{v}^{4}$ (respectively $A^{4}$ ) by

$$
\left.x \rho_{v}(g, h)=\alpha^{-1}\left({ }^{t} g \alpha(x) h\right) \text { (respectively } x \rho(g, h)=\alpha^{-1}\left({ }^{t} g \alpha(x) h\right)\right) .
$$

For $g, h, k \in G_{A}$ and $f \in \mathscr{S}(A)$, put

$$
\Theta_{A}(g ; h, k ; f)=\sum_{x \in Q^{4}} r(g) f(x \rho(h, k)) .
$$

Then (1.8) shows that

$$
\Theta_{\boldsymbol{A}}(\gamma g ; h, k ; f)=\Theta_{\boldsymbol{A}}(g ; h, k ; f)
$$

holds for $r \in G_{Q}$. For a prime $q \neq p$, let $f_{q}$ be the characteristic function of $\boldsymbol{Z}_{q}^{4}$. Define a function $f_{\infty}$ (respectively $f_{p}$ ) on $\boldsymbol{R}^{4}$ (respectively $\boldsymbol{Q}_{p}^{4}$ ) by $f_{\infty}(a, b, c, d)=e^{-p \pi\left(a^{2}+b^{2}+c^{2}+d^{2}\right)} \quad\left(\right.$ respectively $f_{p}(a, b, c, d)=\bar{\chi}(p b) \varphi(a, p b, c, d)$ with the characteristic function $\varphi$ of $\left.Z_{p}^{4}\right)$ and define a function $f$ on $A^{4}$ by $f\left(\left(a_{v}\right),\left(b_{v}\right),\left(c_{v}\right),\left(d_{v}\right)\right)=\prod_{v} f_{v}\left(a_{v}, b_{v}, c_{v}, d_{v}\right)$. Then it is easy to see that the restriction of the function $\Theta_{A}(g ; h, k ; f)$ of $(g, h, k) \in G_{A} \times G_{A} \times G_{A}$ to $G \times G \times G$ is equal to $\Theta(g i, h, k)$. For a subset $A$ in $\boldsymbol{Q}_{p}$, denote the characteristic function of $A$ by $\varphi(; A)$. Then for $f^{(1)}(a, b)=\bar{\chi}(p b) \varphi\left(a ; Z_{p}\right)$ $\varphi\left(b ; 1 / p \boldsymbol{Z}_{p}^{\times}\right)$we have $\tilde{f}^{(1)}(a, b)=\mathscr{F}_{-p, p} f^{(1)}(a, b)=p G(\bar{\chi}) \varphi\left(a ; \boldsymbol{Z}_{p}\right) \chi(-b p) \varphi\left(b ; 1 / p \boldsymbol{Z}_{p}^{\times}\right)$ with $G(\chi)=\int_{Z_{p}} \chi(u) \psi_{p}(u / p) d u$ and for $f^{(2)}(a, b)=\varphi\left(a ; Z_{p}\right) \varphi\left(b ; Z_{p}\right)$ we have $\tilde{f}^{(2)}(a, b)=\mathscr{F}_{p, p} f^{(2)}(a, b)=\varphi\left(a ; Z_{p}\right) \varphi\left(b ; 1 / p Z_{p}\right) . \quad$ For $\sigma=\left(\begin{array}{cc}\alpha & \beta \\ \gamma & \delta\end{array}\right)$ such that $\alpha, \beta, \delta \in \boldsymbol{Z}_{p}, \gamma \in p \boldsymbol{Z}_{p}$ and $\alpha \beta-\gamma \delta=1$, we can easily see that $\lambda(\sigma) \tilde{f}^{(1)}=\chi(\delta) \tilde{f}^{(1)}$ and $\lambda(\sigma) \tilde{f}^{(1)}=\chi(\delta) \tilde{f}^{(1)}$. These imply (1.4) since obviously $\Theta_{A}\left(g k_{q} ; h, k ; f\right)=$ $\Theta_{A}(g ; h, k ; f)$ holds for $k_{q} \in S L\left(2, Z_{q}\right)$ with all primes $q \neq p$. It can easily be verified that

$$
\begin{aligned}
\mathscr{F}_{-p, p}^{-1} \tilde{f}^{(1)}(a, b) & =\int_{Q_{p}} \tilde{f}^{(1)}(x,-a) \psi_{p}(p x b)|p|_{p} d_{p} x \\
& =G(\bar{\chi}) \chi(a p) \varphi\left(a ; 1 / p \boldsymbol{Z}_{p}^{\times}\right) \varphi\left(b ; 1 / p \boldsymbol{Z}_{p}\right)
\end{aligned}
$$


and that

$$
\begin{aligned}
\mathscr{F}_{p, p}^{-1} \tilde{f}^{(2)}(a, b) & =\int_{Q_{p}} \tilde{f}^{(2)}(x,-a) \psi_{p}(-p x b)|p|_{p} d_{p} x \\
& =p^{-1} \varphi\left(a ; 1 / p Z_{p}\right) \varphi\left(b ; 1 / p Z_{p}\right) .
\end{aligned}
$$

Put $f^{\prime}\left(\left(a_{v}\right),\left(b_{v}\right),\left(c_{v}\right),\left(d_{v}\right)\right)=f_{p}^{\prime}\left(a_{p}, b_{p}, c_{p}, d_{p}\right) \prod_{v \neq p} f_{v}\left(a_{v}, b_{v}, c_{v}, d_{v}\right)$ where $f_{p}^{\prime}(a, b$, $c, d)=\chi(p a) \varphi\left(a ; 1 / p Z_{p}\right) \varphi\left(b ; 1 / p Z_{p}\right) \varphi\left(c ; 1 / p Z_{p}\right) \varphi\left(d ; 1 / p Z_{p}\right)$. Then for $\omega=\left(\omega_{v}\right)$ such that $\omega_{\infty}=\left(\begin{array}{cc}0 & 1 \\ -1 & 0\end{array}\right)$ and $\omega_{v}=1$ if $v \neq \infty$ and for $g=\left(g_{v}\right)$ such that $g_{\infty}=\left(\begin{array}{ll}1 & u \\ 0 & 1\end{array}\right)\left(\begin{array}{cc}\sqrt{v} & 0 \\ 0 & \sqrt{v}^{-1}\end{array}\right)$ with $z=u+i v$ and $g_{v}=1$ if $v \neq \infty$, we have

$$
\begin{aligned}
\Theta(-1 / z, h, k) & =\Theta_{A}(\omega g ; h, k ; f)=\Theta_{A}(g \tilde{\omega} ; h, k ; f) \\
& =p^{-2} g(\bar{\chi}) \Theta_{A}\left(g ; h, k ; f^{\prime}\right)=p^{-2} g(\bar{\chi}) \Theta\left(p^{-1} z, h, k\right)
\end{aligned}
$$

with $\tilde{\omega}=\left(\tilde{\omega}_{v}\right)$ where $\tilde{\omega}_{\infty}=1$ and $\tilde{\omega}_{v}=\left(\begin{array}{cc}0 & -1 \\ 1 & 0\end{array}\right)$ for $v \neq \infty$ provided that $h$, $k \in G$. Hence the proof of Proposition 1 is completed.

For $w=x+i y \in H$, put $g_{w}=\left(\begin{array}{ll}1 & x \\ 0 & 1\end{array}\right)\left(\begin{array}{cc}\sqrt{y} & 0 \\ 0 & 1 / \sqrt{y}\end{array}\right)$ and let

$$
\theta\left(z, w_{1}, w_{2}\right)=\Theta\left(z, g_{w_{1}}, g_{w_{2}}\right), \quad \tilde{\theta}\left(z, w_{1}, w_{2}\right)=\tilde{\Theta}\left(z, g_{w_{1}}, g_{w_{2}}\right) .
$$

Denote by $T_{w}^{\chi}(q)$ Hecke operators acting on a space of functions $\varphi(w)$ of $w$ such that $\varphi(\gamma w)=\chi(d) \varphi(w)$ for $\gamma=\left(\begin{array}{ll}a & b \\ c & d\end{array}\right) \in \Gamma$ by the rule

$$
T_{w}^{\chi}(q) \varphi(w)=\sum_{i} \varphi\left(\left(\begin{array}{ll}
1 & 0 \\
0 & q
\end{array}\right) \gamma_{i} w\right) \bar{\chi}\left(d_{i}\right)
$$

where the sum is extended over all $\gamma_{i}=\left(\begin{array}{ll}a_{i} & b_{i} \\ c_{i} & d_{i}\end{array}\right)$ such that $\Gamma\left(\begin{array}{ll}1 & 0 \\ 0 & q\end{array}\right) \Gamma=$ $\bigcup_{i} \Gamma\left(\begin{array}{ll}1 & 0 \\ 0 & q\end{array}\right) r_{i}$ (disjoint).

Let $\varphi$ be as above and $\psi$ is a function satisfying $\psi(\gamma w)=\bar{\chi}(d) \psi(w)$ for $\gamma=\left(\begin{array}{ll}a & b \\ c & d\end{array}\right) \in \Gamma$. Then we have, if the integrals converge,

$$
\int_{\Gamma \backslash H}\left(T_{z}^{\chi}(q) \varphi(z)\right) \psi(z) d_{0} z=\int_{\Gamma \backslash H} \varphi(z)\left(T_{z}^{\chi}(q) * \psi(z)\right) d_{0} z .
$$

Here $T_{z}^{\chi}(q)^{*}$ is the operator defined by $T_{z}^{\chi}(q)^{*} \psi(z)=\sum_{i} \psi\left(\left(\begin{array}{ll}q & 0 \\ 0 & 1\end{array}\right) \sigma_{i} z\right) \chi\left(d_{i}\right)$ where the sum is extended over all $\sigma_{i}=\left(\begin{array}{ll}a_{i} & b_{i} \\ c_{i} & d_{i}\end{array}\right)$ such that $\Gamma\left(\begin{array}{ll}q & 0 \\ 0 & 1\end{array}\right) \Gamma=$ $\bigcup_{i}^{z} \Gamma\left(\begin{array}{ll}q & 0 \\ 0 & 1\end{array}\right) \sigma_{i}$ and $d_{0} z=\frac{d u d v}{v^{2}}$. It is easy to see that $T_{z}^{\chi}(q)^{*}=\chi(q) T_{z}^{\bar{z}}(q)$ if $q \neq p$. 
Proposition 2. For any prime $q$

$$
T_{z}^{*}(q) * \theta\left(z, w_{1}, w_{2}\right)=T_{w_{1}}^{\chi}(q) \theta\left(z, w_{1}, w_{2}\right)=T_{u_{2}}^{\chi}(q) \theta\left(z, w_{1}, w_{2}\right),
$$

whether $q$ equals $p$ or not.

This proposition is almost proved by H. Yoshida ([8]). However we need the exact result convenient to us, so we describe the proof for the sake of completeness and convenience, after $\mathrm{H}$. Yoshida.

Proof. Let the notation be as in the proof of Proposition 1. Put $\tilde{G}=\tilde{G}_{\infty}=G L(2, \boldsymbol{R}), \tilde{G}_{q}=G L\left(2, \boldsymbol{Q}_{q}\right), \tilde{G}_{Q}=G L(2, \boldsymbol{Q})$ and $\tilde{G}_{\boldsymbol{A}}=G L(2, \boldsymbol{A})$. For primes $q \neq p$, put $\tilde{K}_{q}=G L\left(2, Z_{q}\right)$ and $K_{q}=S L\left(2, Z_{q}\right)$. Put

$$
\tilde{K}_{p}=\left\{\gamma=\left(\begin{array}{ll}
a & b \\
c & d
\end{array}\right) \in G L\left(2, Z_{p}\right) \mid c \equiv 0(\bmod p)\right\}
$$

$K_{p}=\tilde{K}_{p} \cap S L\left(2, Z_{p}\right), K_{\infty}=S O(2), B_{v}=\left\{\left(\begin{array}{ll}a & 0 \\ 0 & a\end{array}\right) \mid a \in \boldsymbol{Q}_{v}^{\times}\right\}, B_{Q}=\left\{\left(\begin{array}{ll}a & 0 \\ 0 & a\end{array}\right) \mid a \in \boldsymbol{Q}^{\times}\right\}$ and $B_{A}=\left\{\left(\begin{array}{ll}a & 0 \\ 0 & a\end{array}\right) a \in A^{\times}\right\}$. As usual we can extend the character $\chi$ to the character $\chi_{A}$ on $B_{A} / B_{Q}$ and a function $\varphi$ on $H$ satisfying $\varphi(\gamma z k)=\bar{\chi}(d) \varphi(z)$ $=\bar{\gamma}(d) \varphi(-\bar{z})$ for $\gamma=\left(\begin{array}{ll}a & b \\ c & d\end{array}\right) \in \Gamma_{0}(p)$ to the function $\varphi_{A}$ on $\tilde{G}_{A}$ satisfying

$$
\varphi_{A}\left(\gamma g k_{\infty} k_{p} k_{\jmath}\right)=\mathscr{S}_{A}(g) \chi_{A}(\jmath) \chi_{A}(d)
$$

for $\gamma \in \tilde{G}_{Q}, k_{\infty} \in \tilde{K}_{\infty}=O(2), z \in B_{A}, k \in \prod_{v \neq p, \infty} \tilde{K}_{v}$ and $k_{p}=\left(\begin{array}{ll}a & b \\ c & d\end{array}\right) \in \tilde{K}_{p}$. Let $\varphi$ be as above, $q$ is a prime other than $p$ and put $\psi(z)=\bar{\chi}(q) T_{z}^{\bar{\chi}}(q) \varphi(z)$, then we see that $\psi_{A}(g)=\left(T(q) \psi_{A}\right)(g)=\sum_{i} \varphi_{A}\left(g \beta_{i}\right)$ where the sum is extended over the elements $\beta_{i}$ in $\tilde{G}_{q}$ such that $\tilde{K}_{q}\left(\begin{array}{ll}1 & 0 \\ 0 & q\end{array}\right) \tilde{K}_{q}=\bigcup_{\imath} \beta_{i} \tilde{K}_{q}$ (disjoint). We can extend a function $\Psi$ on $G_{A}$ satisfying

$$
\Psi\left(\gamma g k k_{p}\right)=\Psi(g) \chi_{A}\left(d_{p}\right)
$$

for $\gamma \in G_{Q}, k \in \prod_{v \neq p} K_{v}$ and $k_{p}=\left(\begin{array}{ll}a & b \\ c & d\end{array}\right) \in K_{p}$ to a function $\tilde{\Psi}$ on $\tilde{G}_{\boldsymbol{A}}$ satisfying (1.14) by putting $\tilde{\Psi}\left(\gamma_{g_{\infty}} k \tau\right)=\Psi\left(g_{\infty} k\right) \chi_{A}\left(t_{p}\right)$ for $\gamma \in \tilde{G}_{Q}, g_{\infty} \in G_{\infty}, k \in \prod_{v \neq \infty} K_{v}$, $\tau=\left(\tau_{v}\right) \in \tilde{G}_{\boldsymbol{A}}$ where $\tau_{\infty}=\left(\begin{array}{ll}r & 0 \\ 0 & r\end{array}\right), r>0$ and $\tau_{q}=\left(\begin{array}{ll}1 & 0 \\ 0 & t_{q}\end{array}\right), t_{q} \in \boldsymbol{Z}_{q}^{\times} . \Theta_{\boldsymbol{A}}$ defined in the proof of Proposition 1 is a function on $G_{A} \times G_{A} \times G_{A}$ having the same types of property as (1.15) with respect to each variable so we can extend it to a function $\theta_{A}$ on $\tilde{G}_{A} \times \tilde{G}_{A} \times \tilde{G}_{A}$ which satisfies (1.14) with respect to the first variable and (1.14) modified by replacing $\chi_{A}$ by $\bar{\chi}_{A}$ with respect 
to the second and third variables. Let $g=\left(\begin{array}{ll}1 & u \\ 0 & 1\end{array}\right)\left(\begin{array}{cc}\sqrt{v} & 0 \\ 0 & \sqrt{v}-1\end{array}\right) \in G_{\infty}, h=$ $\left(\begin{array}{ll}1 & x \\ 0 & 1\end{array}\right)\left(\begin{array}{cc}\sqrt{y} & 0 \\ 0 & \sqrt{y}^{-1}\end{array}\right) \in G_{\infty} \quad k \in G_{\infty}, z=u+i v$ and $w=x+i y$. Choose the elements $\beta_{i}$ in $\tilde{G}_{q}$ such that $\tilde{K}_{q}\left(\begin{array}{ll}1 & 0 \\ 0 & q\end{array}\right) \tilde{K}_{q}=\bigcup_{v} \beta_{i} \tilde{K}_{q}$ (disjoint). We can take elements in $\Gamma\left(\begin{array}{ll}1 & 0 \\ 0 & q\end{array}\right) \Gamma$ as such $\beta_{i}$ so that $\Gamma\left(\begin{array}{ll}q & 0 \\ 0 & 1\end{array}\right) \Gamma=\bigcup_{i} \Gamma \alpha_{i}$ with $\alpha_{i}=q \beta_{i}^{-1}$. Note that we can take $\left(\begin{array}{ll}1 & 0 \\ 0 & q\end{array}\right),\left(\begin{array}{ll}q & i \\ 0 & 1\end{array}\right),(i=1,2, \cdots, q)$ as $\beta_{0}, \beta_{i},(i=1,2$, $\cdots, q)$. We denote also by $c_{v^{\prime}}(\gamma)$ an element $\left(g_{v}\right)$ in $\tilde{G}_{\boldsymbol{A}}$ such that $g_{v}=1$ if $v \neq v^{\prime} ; g_{v^{\prime}}=\gamma$ and also by $\delta[\gamma]$ an element $\left(h_{v}\right)$ in $\tilde{G}_{\boldsymbol{A}}$ such that $h_{v}=\gamma$ for all $v$ to avoid confusing $\iota_{v^{\prime}}(\gamma)$ with $\delta[\gamma]$ when $\gamma \in \tilde{G}_{Q^{\prime}}$. We denote by $d(\sigma)$ the lower right entry $d$ of $\sigma=\left(\begin{array}{ll}a & b \\ c & d\end{array}\right)$. Then, by using (1.15), we have

$$
\begin{aligned}
T_{z}^{\chi}(q) & * \theta(z, w, k i) \\
& =\sum_{i} \theta_{A}\left(\iota_{\infty}\left(\alpha_{i} g\right) ; \iota_{\infty}(h), \iota_{\infty}(k) ; f\right) \chi\left(d\left(\alpha_{i}\right)\right) \\
& =\chi(q) \sum_{i} \theta_{A}\left(\iota_{\infty}\left(\beta_{i}^{-1} g\right) ; \iota_{\infty}(h), \iota_{\infty}(k) ; f\right) \chi\left(d\left(\beta_{i}^{-1}\right)\right) \\
& =\chi(q) \sum_{i} \theta_{A}\left(\delta\left[\beta_{i}^{-1}\right] \iota_{\infty}(g) \iota_{q}\left(\beta_{i}\right) ; \iota_{\infty}(h), \iota_{\infty}(k) ; f\right) \\
& =\chi(q) \sum_{i} \theta_{A}\left(\iota_{\infty}(g) \iota_{q}\left(\beta_{i}\right) ; \iota_{\infty}(h), \iota_{\infty}(k) ; f\right) \\
& =\chi(q) \sum_{i} \theta_{A}\left(\delta\left[\left(\begin{array}{cc}
1 & 0 \\
0 & q^{-1}
\end{array}\right)\right] \iota_{\infty}(g) \iota_{q}\left(\beta_{i}\right) ; \iota_{\infty}(h), \iota_{\infty}(k) ; f\right) \\
& =\sum_{i} \theta_{A}\left(\iota_{\infty}\left(\left(\begin{array}{cc}
1 & 0 \\
0 & q^{-1}
\end{array}\right) g\right) \iota_{q}\left(\left(\begin{array}{cc}
1 & 0 \\
0 & q^{-1}
\end{array}\right) \beta_{i}\right) ; \iota_{\infty}(h), \iota_{\infty}(k) ; f\right) \\
& =\sum_{i} \theta_{\boldsymbol{A}}\left(\iota_{\infty}\left(\left(\begin{array}{cc}
q^{1 / 2} & 0 \\
0 & q^{-1 / 2}
\end{array}\right) g\right) \iota_{q}\left(\left(\begin{array}{ll}
1 & 0 \\
0 & q^{-1}
\end{array}\right) \beta_{i}\right) ; \iota_{\infty}(h), \iota_{\infty}(k) ; f\right) \\
& =\theta_{\boldsymbol{A}}\left(\iota_{\infty}(g) ; \iota_{\infty}(h), \iota_{\infty}(k) ; \tilde{f}\right)
\end{aligned}
$$

with $\tilde{f}=q \tilde{f}_{q} \tilde{f}_{\infty} \prod_{v \neq q, \infty} f_{v}$ where $\tilde{f}_{q}=\sum_{i} r_{q}\left(\left(\begin{array}{cc}1 & 0 \\ 0 & q^{-1}\end{array}\right) \beta_{i}\right) f_{q}, \tilde{f}_{\infty}(x)=f_{\infty}(\sqrt{ } \bar{q} x)$ and $f$, $f_{v}$ are the same as in the proof of Proposition 1. We can define the representations, which we also denote by $\rho_{v}$ and $\rho$, of $\tilde{G}_{v} \times \tilde{G}_{v}$ and $\tilde{G}_{\boldsymbol{A}} \times \tilde{G}_{\boldsymbol{A}}$ by (1.9). It is easy to see that

$$
\begin{aligned}
T_{w}^{\chi}(q) & \theta(z, w, k i) \\
& =\sum_{i} \theta_{A}\left(\iota_{\infty}(g) ; \iota_{\infty}(h) \iota_{q}\left(\beta_{i}\right), \iota_{\infty}(k) ; f\right) \\
& =\chi(q) \sum_{i} \theta_{A}\left(\iota_{\infty}(g) ; \iota_{\infty}\left(\left(\begin{array}{ll}
q^{1 / 2} & 0 \\
0 & q^{-1 / 2}
\end{array}\right) h\right) \iota_{q}\left(\left(\begin{array}{ll}
1 & 0 \\
0 & q^{-1}
\end{array}\right) \beta_{i}\right), \iota_{\infty}(k) ; f\right)
\end{aligned}
$$




$$
\begin{aligned}
= & \chi(q) \sum_{i} \sum_{x \ni Q^{4}} r\left(\iota_{\infty}(g)\right) f\left(x \rho\left(\delta\left[\left(\begin{array}{ll}
1 & 0 \\
0 & q
\end{array}\right)\right], 1\right)\right. \\
& \left.\times \rho\left(\iota_{\infty}\left(\left(\begin{array}{cc}
q^{1 / 2} & 0 \\
0 & q^{-1 / 2}
\end{array}\right) h\right), \iota_{\infty}(k)\right) \rho\left(\iota_{q}\left(\left(\begin{array}{ll}
1 & 0 \\
0 & q^{-1}
\end{array}\right) \beta_{i}\right), 1\right)\right) \\
= & \sum_{i} \sum_{x \in Q^{4}} r\left(\iota_{\infty}(g)\right) \\
& \times f\left(x \rho\left(\iota_{\infty}\left(\left(\begin{array}{cc}
q^{1 / 2} & 0 \\
0 & q^{1 / 2}
\end{array}\right) h\right), \iota_{\infty}(k)\right) \rho\left(\iota_{q}\left(\beta_{i}\right), 1\right)\right) \\
= & \theta_{A}\left(\iota_{\infty}(g) ; \iota_{\infty}(h), \iota_{\infty}(k) ; \hat{f}\right)
\end{aligned}
$$

with $\hat{f}=\tilde{f}_{\infty} \hat{f}_{q} \prod_{v \neq q, \infty} f_{v}$ where $\hat{f}_{q}(x)=\sum_{i} f_{q}\left(x \rho_{q}\left(\beta_{i}, 1\right)\right)$. Therefore it is sufficient to show that $q \tilde{f}_{q}=\hat{f}_{q}$ in order to prove the former part of Proposition 2 in case $q \neq p$. Choose the elements $\delta_{i} \in \Gamma$ such that $\Gamma\left(\begin{array}{ll}p & 0 \\ 0 & 1\end{array}\right) \Gamma=$ $\bigcup_{i} \Gamma\left(\begin{array}{ll}p & 0 \\ 0 & 1\end{array}\right) \delta_{i}$ (disjoint). Then it holds $K_{p}\left(\begin{array}{ll}1 & 0 \\ 0 & p\end{array}\right) K_{p}=\bigcup_{\imath} \delta_{i}^{-1}\left(\begin{array}{ll}1 & 0 \\ 0 & p\end{array}\right) K_{p}$ and we have

$$
\begin{aligned}
T_{z}^{\chi}(p) & * \theta(z, w, k i) \\
& =\sum_{i} \theta_{A}\left(\iota_{\infty}\left(\left(\begin{array}{ll}
p & 0 \\
0 & 1
\end{array}\right) \delta_{i} g\right) ; \iota_{\infty}(h), \iota_{\infty}(k) ; f\right) \chi\left(d\left(\delta_{i}\right)\right) \\
& =\sum_{i} \theta_{A}\left(\iota_{\infty}\left(\left(\begin{array}{cc}
1 & 0 \\
0 & 1 / p
\end{array}\right) \delta_{i} g\right) ; \iota_{\infty}(h), \iota_{\infty}(k) ; f\right) \chi\left(d\left(\delta_{i}\right)\right) \\
& =\sum_{i} \theta_{A}\left(\iota_{\infty}(g) \iota_{p}\left(\delta_{i}^{-1}\left(\begin{array}{ll}
1 & 0 \\
0 & p
\end{array}\right)\right) ; \iota_{\infty}(h), \iota_{\infty}(k) ; f\right) \chi\left(d\left(\delta_{i}\right)\right) \\
& =\sum_{i} \theta_{A}\left(\delta\left[\left(\begin{array}{cc}
1 & 0 \\
0 & p^{-1}
\end{array}\right)\right] \iota_{\infty}(g) \iota_{p}\left(\delta_{i}^{-1}\left(\begin{array}{ll}
1 & 0 \\
0 & p
\end{array}\right)\right) ; \iota_{\infty}(h), \iota_{\infty}(k) ; f\right) \chi\left(d\left(\delta_{i}\right)\right) \\
& =\sum_{i} \theta_{A}\left(\iota_{\infty}\left(\left(\begin{array}{cc}
1 & 0 \\
0 & p^{-1}
\end{array}\right) g\right) \iota_{p}\left(\left(\begin{array}{cc}
1 & 0 \\
0 & p^{-1}
\end{array}\right) \delta_{i}^{-1}\left(\begin{array}{ll}
1 & 0 \\
0 & p
\end{array}\right)\right) ; \iota_{\infty}(h), \iota_{\infty}(k) ; f\right) \chi\left(d\left(\delta_{i}\right)\right) \\
& =\sum_{i} \theta_{A}\left(\iota_{\infty}\left(\left(\begin{array}{cc}
p^{1 / 2} & 0 \\
0 & p^{-1 / 2}
\end{array}\right) g\right) \iota_{p}\left(\left(\begin{array}{ll}
1 & 0 \\
0 & p^{-1}
\end{array}\right) \delta_{i}^{-1}\left(\begin{array}{ll}
1 & 0 \\
0 & p
\end{array}\right)\right) ; \iota_{\infty}(h), \iota_{\infty}(k) ; f\right) \chi\left(d\left(\delta_{i}\right)\right) \\
& =\theta_{A}\left(\iota_{\infty}(g) ; \iota_{\infty}(h), \iota_{\infty}(k) ; \tilde{f}\right)
\end{aligned}
$$

with $\tilde{f}=p \tilde{f}_{p} \tilde{f}_{\infty} \prod_{v \neq p, \infty} f_{v}$ where $\tilde{f}_{p}=\sum_{i} \chi\left(d\left(\delta_{i}\right)\right) r_{p}\left(\left(\begin{array}{ll}1 & 0 \\ 0 & p^{-1}\end{array}\right) \delta_{i}^{-1}\left(\begin{array}{ll}1 & 0 \\ 0 & p\end{array}\right)\right) f_{p}, \quad \tilde{f}_{\infty}(x)$ $=f_{\infty}(\sqrt{p} x)$ and $f, f_{v}$ are the same as in the proof of Proposition 1. Choose the elements $\gamma_{i} \in \Gamma$ such that $\Gamma\left(\begin{array}{ll}1 & 0 \\ 0 & p\end{array}\right) \Gamma=\bigcup_{i} \Gamma\left(\begin{array}{ll}1 & 0 \\ 0 & p\end{array}\right) \gamma_{i}$ (disjoint). Then it holds $K_{p}\left(\begin{array}{ll}p & 0 \\ 0 & 1\end{array}\right) K_{p}=\bigcup_{\imath} \gamma_{i}^{-1}\left(\begin{array}{ll}p & 0 \\ 0 & 1\end{array}\right) K_{p}$ and we have 
$T_{w}^{\chi}(p) \theta(z, w, k i)$

$$
\begin{aligned}
= & \sum_{i} \theta_{A}\left(\iota_{\infty}(g) ; \iota_{\infty}\left(\left(\begin{array}{cc}
1 & 0 \\
0 & p
\end{array}\right) \gamma_{i} h\right), \iota_{\infty}(k) ; f\right) \bar{\chi}\left(d\left(\gamma_{i}\right)\right) \\
= & \sum_{i} \theta_{A}\left(\iota_{\infty}(g) ; \iota_{\infty}(h) \iota_{p}\left(\gamma_{i}^{-1}\left(\begin{array}{ll}
p & 0 \\
0 & 1
\end{array}\right)\right), \iota_{\infty}(k) ; f\right) \bar{\chi}\left(d\left(\gamma_{i}\right)\right) \\
= & \sum_{\imath} \theta_{A}\left(\iota_{\infty}(g) ; \iota_{\infty}\left(\left(\begin{array}{cc}
p^{-1 / 2} & 0 \\
0 & p^{1 / 2}
\end{array}\right) h\right) \iota_{p}\left(\left(\begin{array}{cc}
p^{-1} & 0 \\
0 & 1
\end{array}\right) \gamma_{i}^{-1}\left(\begin{array}{ll}
p & 0 \\
0 & 1
\end{array}\right)\right), \iota_{\infty}(k) ; f\right) \\
& \times \bar{\chi}\left(d\left(\gamma_{i}\right)\right) \\
= & \sum_{i} \sum_{x \in Q^{4}} r_{\infty}\left(\iota_{\infty}(g)\right) \bar{\chi}\left(d\left(\gamma_{i}\right)\right) f\left(x \rho\left(\delta\left[\left(\begin{array}{ll}
p & 0 \\
0 & 1
\end{array}\right)\right], 1\right)\right. \\
& \left.\times \rho\left(\iota_{\infty}\left(\left(\begin{array}{cc}
p^{-1 / 2} & 0 \\
0 & q^{1 / 2}
\end{array}\right) h\right), \iota_{\infty}(k)\right) \rho\left(\iota_{p}\left(\left(\begin{array}{cc}
p^{-1} & 0 \\
0 & 1
\end{array}\right) \gamma_{i}^{-1}\left(\begin{array}{ll}
p & 0 \\
0 & 1
\end{array}\right)\right), 1\right)\right) \\
= & \sum_{i} \sum_{x \in Q^{4}} r_{\infty}\left(\iota_{\infty}(g)\right) \bar{\chi}\left(d\left(\gamma_{i}\right)\right) \\
& \times f\left(x \rho\left(\iota_{\infty}\left(\left(\begin{array}{cc}
p^{1 / 2} & 0 \\
0 & p^{1 / 2}
\end{array}\right) h\right), \iota_{\infty}(k)\right) \rho\left(\iota_{p}\left(\gamma_{i}^{-1}\left(\begin{array}{ll}
p & 0 \\
0 & 1
\end{array}\right)\right), 1\right)\right) \\
= & \theta_{A}\left(\iota_{\infty}(g) ; \iota_{\infty}(h), \iota_{\infty}(k) ; \hat{f}\right)
\end{aligned}
$$

with $\hat{f}=\tilde{f}_{\infty} \hat{f}_{p} \prod_{v \neq p, \infty} f_{v}$ where $\tilde{f}_{p}(x)=\sum_{i} \bar{\chi}\left(d\left(\gamma_{i}\right)\right) f_{p}\left(x \rho_{p}\left(\gamma_{i}^{-1}\left(\begin{array}{ll}p & 0 \\ 0 & 1\end{array}\right), 1\right)\right)$, Therefore it is sufficient to show that $p \tilde{f}_{p}=f_{p}$ in order to prove the former part of Proposition 2 in case $q=p$. Since we can take $\left(\begin{array}{cc}1 & 0 \\ -p i & 1\end{array}\right)(i=0, \cdots, p-1)$ and $\left(\begin{array}{rr}1 & -i \\ 0 & 1\end{array}\right),(i=0, \cdots, p-1)$ as $\delta_{i}$ and $\gamma_{i}$ respectively, it is sufficient to show that

$$
\sum_{i=0}^{p-1} f_{p}\left(x \rho_{p}\left(\left(\begin{array}{ll}
1 & i \\
0 & 1
\end{array}\right)\left(\begin{array}{ll}
p & 0 \\
0 & 1
\end{array}\right), 1\right)\right)=\sum_{i=0}^{p-1} p r_{p}\left(\left(\begin{array}{ll}
1 & 0 \\
i & 1
\end{array}\right)\right) f_{p}(x) \quad\left(=p \tilde{f}_{p}(x)\right)
$$

for all $x \in \boldsymbol{Q}_{p}^{4}$.

Let the notation be as in the proof of Proposition 1. Then $f_{p}(a, b, c$, $d)=f^{(1)}(a, b) f^{(2)}(c, d)$ and $r_{p}(g) f_{p}=r_{p, 1}(g) f^{(1)} r_{p, 2}(g) f^{(2)}$. Therefore we compute $r_{p, i}(g) f^{(\imath)},(i=1,2)$. Since $r_{p, 1}(g)=\mathscr{F}_{-p, p}^{-1} \lambda(g) \mathscr{F}_{-p, p}$ and

$$
\lambda\left(\begin{array}{ll}
1 & 0 \\
i & 1
\end{array}\right) \tilde{f}^{(1)}(a, b)=p G(\bar{\chi}) \varphi\left(a+i b ; Z_{p}\right) \varphi\left(b ; 1 / p Z_{p}\right) \chi_{A}(-b p),
$$

we have

$$
r_{p, 1}\left(\left(\begin{array}{ll}
1 & 0 \\
i & 1
\end{array}\right)\right) f^{(1)}(a, b)=G(\bar{\chi}) \int_{1 / p Z_{p}^{\times}} \varphi\left(a+i u ; Z_{p}\right) \chi_{A}(p u) d u
$$




$$
=G(\bar{\chi}) \varphi\left(a ; 1 / p Z_{p}^{\times}\right) \varphi\left(b ; 1 / p Z_{n}\right) \chi_{A}\left(-p i^{-1} a\right) \psi\left(-p i^{-1} a b\right)
$$

if $i \neq 0$. Recall that

$$
r_{p, 2}(g)=\mathscr{F}_{p, p}^{-1} \lambda(g) \mathscr{F}_{p, p} \quad \text { and } \quad \tilde{f}^{(2)}(c, d)=\varphi\left(c ; Z_{p}\right) \varphi\left(d ; 1 / p Z_{p}\right),
$$

then we have

$$
\lambda\left(\left(\begin{array}{ll}
1 & 0 \\
i & 1
\end{array}\right)\right) \tilde{f}^{(2)}(c, d)=\varphi\left(c+i d ; Z_{p}\right) \varphi\left(d ; 1 / p Z_{p}\right)
$$

and

$$
r_{p, 2}\left(\begin{array}{ll}
1 & 0 \\
i & 0
\end{array}\right) \tilde{f}_{p}^{(2)}(c, d)=p^{-1} \psi\left(p i^{-1} c d\right) \varphi\left(c ; 1 / p Z_{p}\right) \varphi\left(d ; 1 / p Z_{p}\right)
$$

if $i \neq 0$. Hence we have

$$
\begin{aligned}
p \tilde{f}_{p}(a, & b, c, d)=p\left(\varphi\left(a ; Z_{p}\right) \varphi\left(b ; 1 / p Z_{p}\right) \varphi\left(c ; Z_{p}\right) \varphi\left(d ; Z_{p}\right) \bar{\chi}_{A}(p b)\right. \\
& +\sum_{k=1}^{p-1} p^{-1} G(\bar{\chi}) \psi(p k(c d-a b)) \chi_{A}(-p k a) \\
& \times \varphi\left(c ; 1 / p Z_{p}\right) \varphi\left(d ; 1 / p Z_{p}\right) \varphi\left(a ; 1 / p Z_{p}^{\times}\right) \varphi\left(b ; 1 / p Z_{p}\right) .
\end{aligned}
$$

We can easily see that $\hat{f}_{p}(a, b, c, d)=0$ unless $b \in 1 / p Z_{p}$ and that

$$
\begin{aligned}
& \hat{f}_{p}(a, b, c, d)=p \bar{\chi}(p b) \quad \text { if } b \in 1 / p Z_{p}^{\times}, d \in Z_{p}, a \in Z_{p}, c \in Z_{p}, \\
& \hat{f}_{p}(a, b, c, d)=\bar{\chi}(p b) \quad \text { if } b \in 1 / p \boldsymbol{Z}_{p}^{\times}, d \in \boldsymbol{Z}_{p}, a \in 1 / p \boldsymbol{Z}_{p}^{\times}, c \in 1 / p \boldsymbol{Z}_{p} \text {, } \\
& \hat{f}_{p}(a, b, c, d)=\bar{\chi}(p a) \bar{\chi}\left(p^{2}(a b-c d)\right) \text { if } b \in 1 / p \boldsymbol{Z}_{p}, d \in 1 / p \boldsymbol{Z}_{p}^{\times}, a \in 1 / p \boldsymbol{Z}_{p}^{\times}, \\
& \hat{f}_{p}(a, b, c, d)=0 \quad \text { otherwise. }
\end{aligned}
$$

Using this, it is easy to see that $p \tilde{f}_{p}(x)=\hat{f}_{p}(x)$ in each case stated above. On the other hand, since we have

$$
\begin{aligned}
& q \tilde{f}_{q}(a, b, c, d)=q\left(r_{q}\left(\left(\begin{array}{ll}
1 & 0 \\
0 & 1
\end{array}\right)\right)+\sum_{i=1}^{q} r_{q}\left(\left(\begin{array}{cc}
q & i \\
0 & q^{-1}
\end{array}\right)\right)\right) f_{q}(a, b, c, d) \\
& =q f_{q}(a, b, c, d)+q^{-1} \sum_{i=1}^{q} \psi_{q}(p(b c-a d) q i) f_{q}(q a, q b, q c, q d)
\end{aligned}
$$

for $q \neq p$, we can easily verify $q \tilde{f}_{q}(x)=\hat{f}_{q}(x)$ case by case. These complete the proof of Proposition 2.

We call a function $\varphi$ satisfying following conditions on $H$ an automorphic wave form with character $\chi$ :

1. For $z=u+i v \in H, \varphi(z)$ is an eigenfunction of $D_{z}=v^{2}\left(\frac{\partial^{2}}{\partial u^{2}}+\frac{\partial^{2}}{\partial v^{2}}\right)$, 
i.e., $D_{z} \varphi=\lambda \varphi$ with $\lambda \in C$.

2. $\varphi(\gamma z)=\chi(d) \varphi(z)$ for all $\gamma=\left(\begin{array}{ll}a & b \\ c & d\end{array}\right) \in \Gamma$.

3. $\varphi(u+i v)=O\left(v^{k}\right), v \rightarrow \infty$, with a constant $\kappa \in \boldsymbol{R}$, uniformly in $u$. Put $\rho=(1+\sqrt{1+4 \lambda}) / 2, \nu=\sqrt{1 / 4+\lambda}$. Let $u_{j}$ be a representative of a $\Gamma$ equivalence class of cusps and $\sigma_{\jmath}$ is an element of $S L(2, Z)$ such that $\sigma_{j} \infty=u_{j}$. Then $\varphi$ has the following Fourier expansion:

$$
\varphi\left(\sigma_{j} z\right)=a^{(j)} v^{\rho}+b^{(j)} v^{1-\rho}+\sum_{n \neq 0} a_{n}^{(j)} v^{1 / 2} K_{\nu}\left(2 \pi|n| v / N_{j}\right) e^{2 \pi n i u / N_{j}}
$$

with the modified Bessel function $K_{\nu}$ and an appropriate integer $N_{j}$. Let $u_{0}=\infty$ and $\sigma_{0}=\left(\begin{array}{ll}1 & 0 \\ 0 & 1\end{array}\right)$, and put $a_{n}^{(0)}=a_{n}$. Then $N_{0}=1$. We call $\varphi$ a cusp form if $a^{(j)}=b^{(j)}=0$ for all $j$. For simplicity, we assume $\varphi(-\bar{z})=\varphi(z)$ which means $a_{n}=a_{-n}$ for all $n$. Notice that a cusp form which is a common eigenfunction for all Hecke operators is the sum of two common eigenfunctions $\varphi_{+}, \varphi_{-}$such that $\varphi_{+}(-\bar{z})=\varphi_{+}(z), \quad \varphi_{-}(-\bar{z})=-\varphi_{-}(z)$. We further assume $a_{1}=a_{-1}=1$. Define

$$
F\left(w_{1}, w_{2}\right)=\int_{\Gamma \backslash H} \varphi(z) \theta\left(z, w_{1}, w_{2}\right) d_{0} z
$$

where $d_{0} z=v^{-2} d u d v$ for $z=u+i v$. The following proposition guarantees that $F$ is an eigenfunction of $D_{w_{1}}, D_{w_{2}}$.

Proposition 2.

$$
D_{z} \theta\left(z, w_{1}, w_{2}\right)=D_{w_{1}} \theta\left(z, w_{1}, w_{2}\right)=D_{w_{2}} \theta\left(z, w_{1}, w_{2}\right) .
$$

We can easily prove this in the same way as in the proof of [7], Lemma 1.5. Thus we skip the proof.

By Proposition 1, Proposition 2 and the additional assumptions, we obtain the Fourier expansion of $F$, and can easily see the equality

$$
F\left(w_{1}, w_{2}\right)=c \varphi\left(w_{1}\right) \varphi\left(w_{2}\right)
$$

with a certain constant $c$ which can not be determined by these Propositions and assumptions. Therefore our next task is to determine the constant $c$. For this purpose, we consider the integral

$$
\int_{0}^{\infty} \int_{0}^{1} F(w, w) d x y^{s-1} d y \quad(w=x+i y) .
$$

On one hand, for $\varphi(w)=\sum_{n \neq 0} a_{|n|} y^{1 / 2} K_{\nu}(2 \pi|n| y) e^{2 \pi n i x}$, it holds 


$$
\begin{aligned}
\int_{0}^{\infty} \int_{0}^{1} F(w, w) d x y^{s-1} d y=c \int_{0}^{\infty} \int_{0}^{1} \varphi(w) \varphi(w) d x y^{s-1} d y \\
=2 c\left(\sum_{n \geq 1} a_{n}^{2} n^{-(s+1)}\right)(2 \pi)^{-(s+1)} \int_{0}^{\infty} K_{\nu}(y)^{2} y^{s-1} d y \\
=2 c L(s+1, \chi)\left(\sum_{n \geq 1} a_{n}^{2} n^{-(s+1)}\right) \\
\quad \times(2 \pi)^{-(s+1)} \int_{0}^{\infty} K_{\nu}(y)^{2} y^{s-1} d y
\end{aligned}
$$

(see [1]) with $L(s, \chi)=\sum_{n=1}^{\infty} \chi(n) n^{-s}$. On the other hand, substituting the right-hand side of (1.17) for $F(w, w)$ in (1.20) and exchanging the order of integrations, we obtain

$$
\begin{aligned}
& \int_{0}^{\infty} \int_{0}^{1} F(w, w) d x y^{s-1} d y \\
&=\int_{\Gamma \backslash H} \varphi(z) \int_{0}^{\infty} \int_{0}^{1} \theta(z, w, w) y^{s-1} d x d y d_{0} z, \quad(w=x+i y) .
\end{aligned}
$$

We can easily justify this formal computation by the absolute convergence of the integrals for sufficient large Res. It is more convenient to consider, instead of $\int_{0}^{\infty} \int_{0}^{1} \theta(z, w, w) y^{s-1} d x d y$, another integral

$$
E_{I}(z, s)=\int_{0}^{\infty} \int_{0}^{1} \tilde{\theta}(z, w, w) y^{s-1} d x d y .
$$

Now recall the definition (1.2) and (1.13) of $\tilde{\Theta}\left(z, g_{1}, g_{2}\right)$ and $\tilde{\theta}\left(z, w_{1}, w_{2}\right)$, then we see that an element $\left(\begin{array}{ll}a & b \\ c & d\end{array}\right)$ of $L$ in (1.2) is uniquely written as the sum of two elements $\left(\begin{array}{cc}a & e / 2 \\ e / 2 & d\end{array}\right),\left(\begin{array}{cc}0 & f / 2 \\ -f / 2 & 0\end{array}\right)$ where $e$ and $f$ are integers with the same parity. Since ${ }^{t} g\left(\begin{array}{rr}0 & 1 \\ -1 & 0\end{array}\right) g=\left(\begin{array}{rr}0 & 1 \\ -1 & 0\end{array}\right)$ for $g \in G$, we can decompose the summation defining $\tilde{\Theta}\left(z, g_{1}, g_{2}\right)$ into two parts, only when $g_{1}=g_{2}$, as follows.

$$
\tilde{\Theta}(z, g, g)=v^{1 / 2} \overline{\theta(z)} \Theta_{3}(z, g)+v^{1 / 2} \overline{\theta_{0}(z)} \Theta_{3,0}(z, g)
$$

where

$$
\begin{aligned}
\theta(z) & =\sum_{n \in Z} e^{2 \pi i n^{2} z}, \quad \theta_{0}(z)=\sum_{n \in \boldsymbol{Z}, n ; \text { odd }} e^{2 \pi i n^{2} z / 4}, \\
\Theta_{3}(z, g) & =\sqrt{v} \sum_{X=\left(\begin{array}{c}
a \\
b \\
b
\end{array}\right) \in L_{3}} \chi(a) e^{2 \pi i\left(-u \operatorname{det} X+i 2-1 v \operatorname{tr}\left(\langle t g X g)^{2}\right\rangle\right)}
\end{aligned}
$$

and 


$$
\Theta_{3,0}(z, g)=\sqrt{v} \sum_{X=\left(\begin{array}{c}
a \\
b \\
b
\end{array}\right) \in L_{3}, 0} \chi(a) e^{2 \pi i\left(-u \operatorname{det} X+i 2-1 v \operatorname{tr}\left(\langle t g X g)^{2}\right\rangle\right)},
$$

with $L_{3}=\left\{\left(\begin{array}{ll}a & b \\ b & c\end{array}\right) \mid a, b, c \in Z\right\}$ and $L_{3,0}=\left\{\left(\begin{array}{cc}a & b / 2 \\ b / 2 & c\end{array}\right) \mid a, b, c \in Z, b\right.$; odd $\}$.

Therefore we obtain

$$
\begin{aligned}
E_{I}(z, s) & =\sqrt{v} \overline{\theta(z)} \int_{0}^{\infty} \int_{0}^{1} \Theta_{3}\left(z,\left(\begin{array}{ll}
1 & x \\
0 & 1
\end{array}\right)\left(\begin{array}{cc}
\sqrt{y} & 0 \\
0 & 1 / \sqrt{y}
\end{array}\right)\right) d x y^{s-1} d y \\
+ & \sqrt{v} \overline{\theta_{0}(z)} \int_{0}^{\infty} \int_{0}^{1} \Theta_{3,0}\left(z,\left(\begin{array}{ll}
1 & x \\
0 & 1
\end{array}\right)\left(\begin{array}{cc}
\sqrt{y} & 0 \\
0 & 1 / \sqrt{y}
\end{array}\right)\right) d x y^{s-1} d y .
\end{aligned}
$$

We have to make explicit computation of two integrals in (1.28) to show that they are equal to Siegel's generalizations of Eisenstein-Epstein series. Put

$$
\begin{aligned}
& E_{\theta}(z, s)=\int_{0}^{\infty} \int_{0}^{1} \Theta_{3}\left(z,\left(\begin{array}{ll}
1 & x \\
0 & 1
\end{array}\right)\left(\begin{array}{cc}
\sqrt{y} & 0 \\
0 & 1 / \sqrt{y}
\end{array}\right)\right) d x y^{s-1} d y, \\
& E_{\theta, 0}(z, s)=\int_{0}^{\infty} \int_{0}^{1} \Theta_{3,0}\left(z,\left(\begin{array}{ll}
1 & x \\
0 & 1
\end{array}\right)\left(\begin{array}{cc}
\sqrt{y} & 0 \\
0 & 1 / \sqrt{y}
\end{array}\right)\right) d x y^{s-1} d y .
\end{aligned}
$$

We treat only the first integral here because the second integral is reduced to the first. $E_{\theta}(z, s)$ can be transformed to the following Fourier series in $z$.

$$
\begin{aligned}
& E_{\theta}(z, s)=\sqrt{v} \sum_{n \in \boldsymbol{Z}} e^{2 \pi i n u} \int_{0}^{\infty} \int_{0}^{1} y^{s-1} \sum_{X=\left(\begin{array}{ll}
a & b \\
b & c
\end{array}\right) \in L_{3},-\operatorname{det} X=n} \chi(a) \\
& \left.\times \exp \left(-\pi v \operatorname{tr}\left(\left(\left(\begin{array}{cc}
\sqrt{y} & 0 \\
0 & 1 / \sqrt{y}
\end{array}\right)\left(\begin{array}{ll}
1 & 0 \\
x & 1
\end{array}\right) X\left(\begin{array}{cc}
1 & x \\
0 & 1
\end{array}\right)\left(\begin{array}{cc}
\sqrt{y} & 0 \\
0 & 1 / \sqrt{y}
\end{array}\right)\right)^{2}\right)\right)\right) d x d y .
\end{aligned}
$$

Since $a \neq 0$ in (1.31) and $\left(\begin{array}{ll}1 & 0 \\ m & 1\end{array}\right)\left(\begin{array}{ll}a & b \\ b & c\end{array}\right)\left(\begin{array}{ll}1 & m \\ 0 & 1\end{array}\right)=\left(\begin{array}{cc}a & a m+b \\ a m+b & a m^{2}+2 b m+c\end{array}\right)$ $\in L_{3}$ for $m \in Z$ if $\left(\begin{array}{ll}a & b \\ b & c\end{array}\right) \in L_{3}$, we can extend the interval of the integration with respect to $x$ in (1.31) by letting $b$ run over representatives modulo $a$ instead of letting $b$ run over all integers in the summation of (1.31). Therefore we obtain

$$
\begin{aligned}
E_{\theta}(z, s)=\sqrt{v} \sum_{n \in \mathbf{Z}} e^{2 \pi i n u} \int_{0}^{\infty} \int_{-\infty}^{\infty} \sum_{\substack{a \neq 0 \\
a \in \boldsymbol{Z}}} \sum_{\substack{b \bmod a, b \geqq n \bmod a}} \chi(a) y^{s-1} \\
\times \exp \left(-\pi v \operatorname{tr}\left(\left(\left(\begin{array}{cc}
\sqrt{y} & 0 \\
0 & 1 / \sqrt{y}
\end{array}\right)\left(\begin{array}{cc}
1 & 0 \\
x & 1
\end{array}\right)\left(\begin{array}{cc}
a & b \\
b & \left(b^{2}-n\right) / a
\end{array}\right)\left(\begin{array}{cc}
1 & x \\
0 & 1
\end{array}\right)\right.\right.\right. \\
\left.\left.\left.\left.\quad \times\left(\begin{array}{cc}
\sqrt{y} & 0 \\
0 & 1 / \sqrt{y}
\end{array}\right)\right)^{2}\right)\right)\right) d x d y .
\end{aligned}
$$


Change the variable $x$ to $x-b / a$, then the summand of the sum in the above integral becomes independent of $b$ and the signature of $a$. Hence we have

$$
\begin{aligned}
& E_{\theta}(z, s)=2 \sqrt{v} \sum_{n \in Z} e^{2 \pi i n u} \sum_{\substack{a>0 \\
a \in Z}} \chi(a) A_{a}(n) \int_{0}^{\infty} \int_{-\infty}^{\infty} y^{s-1} \\
& \times \exp \left(-\pi v \operatorname{tr}\left(\left(\left(\begin{array}{cc}
\sqrt{y} & 0 \\
0 & 1 / \sqrt{ } y
\end{array}\right)\left(\begin{array}{cc}
1 & 0 \\
x & 1
\end{array}\right)\left(\begin{array}{cc}
a & 0 \\
0 & -n / a
\end{array}\right)\left(\begin{array}{cc}
1 & x \\
0 & 1
\end{array}\right)\left(\begin{array}{rr}
\sqrt{y} & 0 \\
0 & 1 / \sqrt{ } y
\end{array}\right)\right)^{2}\right)\right) \\
& \times d x d y,
\end{aligned}
$$

where we denote by $A_{a}(n)$ the number of solutions modulo $a$ for the equation $x^{2} \equiv n(\bmod a)$. Next, change the variables $y, x$ to $a^{-1} y, a^{-1} x$ or $|n|^{1 / 2} a^{-1} y,|n|^{1 / 2} a^{-1} x$ according as $n$ is equal to 0 or not. Then we have

$$
\begin{aligned}
E_{\theta}(z, s) & =2 \sqrt{v} \Xi(s, 0) W_{0}(s, \pi v) \\
+ & 2 \sqrt{v} \sum_{\substack{n \in Z \\
n \neq 0}} e^{2 \pi i n u}|n|^{(s+1) / 2} \Xi(s, n) W_{-\operatorname{sgn} n}(s, \pi|n| v)
\end{aligned}
$$

where

$$
\begin{gathered}
\Xi(s, n)=\sum_{n=1}^{\infty} \chi(a) A_{a}(n) a^{-(s+1)} \\
W_{\varepsilon}(s, v)=\int_{0}^{\infty} \int_{-\infty}^{\infty} y^{s-1} \\
\times \exp \left(-\pi v \operatorname{tr}\left(\left(\left(\begin{array}{cc}
\sqrt{y} & 0 \\
0 & 1 / \sqrt{ } \bar{y}
\end{array}\right)\left(\begin{array}{ll}
1 & 0 \\
x & 1
\end{array}\right)\left(\begin{array}{ll}
1 & 0 \\
0 & \varepsilon
\end{array}\right)\left(\begin{array}{cc}
1 & x \\
0 & 1
\end{array}\right)\left(\begin{array}{cc}
\sqrt{y} & 0 \\
0 & 1 / \sqrt{ } \bar{y}
\end{array}\right)\right)^{2}\right)\right) d x d y
\end{gathered}
$$

and $\operatorname{sgn} n=n /|n|$.

LEMMA 1.

$$
\begin{aligned}
W_{-\operatorname{sgn} n}(s, \pi|n| v)= & 2^{s / 2-1} \Gamma((s+1) / 2)(2 \pi v)^{-(s+1) / 2} \\
& \rho_{n}(s+1, v)\left\{\begin{array}{cc}
|n|^{-(s+1) / 2} & (n \neq 0), \\
1 & (n=0),
\end{array}\right.
\end{aligned}
$$

where

$$
\rho_{n}(s, v)=\int_{-\infty}^{\infty} \frac{e^{-2 \pi i n v \xi} d \xi}{(1-i \xi)^{(s+1) / 2}(1+i \xi)^{s / 2}} .
$$

Proof. By the definition we can express $W_{\varepsilon}(v)$ as an integral hof the modified Bessel function, namely,

$$
W_{\varepsilon}(s, v)=1 / 2 \int_{0}^{\infty} \sqrt{x^{-2 v x}} \int_{0}^{\infty} e^{-v\left(y+\left(x_{x}+\varepsilon\right)^{2 / y}\right)} y^{s / 2-1} d y d x
$$




$$
=\int_{0}^{\infty}|\varepsilon+x|^{s / 2} e^{-2 v x} K_{-s / 2}(2 v|x+\varepsilon|) \sqrt{x} d x .
$$

Using another integral expression

$$
\begin{aligned}
\mid \varepsilon+ & \left.x\right|^{s / 2} K_{-s / 2}(2 v|x+\varepsilon|) \\
& =v^{-s / 2} 2^{-1} \pi^{-1 / 2} \Gamma(s / 2+1 / 2) \int_{-\infty}^{\infty} \frac{e^{-i \xi(2 v x+2 v \varepsilon)}}{\left(\xi^{2}+1\right)^{s / 2+1 / 2}} d \xi
\end{aligned}
$$

for $K_{-s / 2}$ and changing the order of the integrations, we have

$$
\begin{aligned}
& W_{\varepsilon}(s, v)=v^{-s / 2} 2^{-1} \pi^{-1 / 2} \Gamma(s / 2+1 / 2) \\
& \quad \times \int_{-\infty}^{\infty} \frac{e^{-i 2 \xi v \varepsilon}}{\left(\xi^{2}+1\right)^{s / 2+1 / 2}} d \xi \int_{0}^{\infty} \sqrt{x} e^{-2 v x(1+i \xi)} d x
\end{aligned}
$$

By changing the variable $x$ to $x / 2 v(1+i \xi)$ and rotating the path of integration to let it go back to the real axis, we have

$$
\begin{aligned}
& \int_{0}^{\infty} \sqrt{x} e^{-2 v x(1+i \xi)} d x=\sqrt{2 v(1+i \xi)^{-1}} \int_{0}^{2 v(1+i \xi)} \sqrt{x} e^{-x} d x \\
&=\sqrt{2 v(1+i \xi) \pi} \\
&-1
\end{aligned}
$$

Hence we obtain

$$
W_{\varepsilon}(s, v)=v^{-s / 2} 2^{-3 / 2} \Gamma^{\prime}(s / 2+1 / 2) \int_{-\infty}^{\infty} \frac{e^{i 2 \xi v \varepsilon}}{\left(\xi^{2}+1\right)^{s / 2+1 / 2}(1-i \xi)^{1 / 2}} d \xi
$$

by changing the variable $\xi$ to $-\xi$, which completes the proof.

Lemma 1 shows that

$$
E_{\theta}(z, s)=2^{s / 2} \Gamma((s+1) / 2)(2 \pi v)^{-(s+1) / 2} \sqrt{v} \sum_{n \in Z} e^{2 \pi i n u} \rho_{n}(s+1, v) \Xi(s, n) .
$$

2. After Siegel we define generalizations of Eisenstein-Epstein series, which we simply call Eisenstein series in this paper, and compute their Fourier expansions. For integers $a, c$ such that $(a, c)=1$ and $c>0$, put

$$
\lambda_{1}(a / c)=2^{-1} \sqrt{i / c} \sum_{h=1}^{2 c} e^{\pi i h^{2} a / c},
$$

then we obtain

LEMMa 2. Let $(-), \varepsilon_{d}$ be as in [2], namely $\left(\frac{a}{b}\right)=\left(\frac{a}{|b|}\right)_{J}(a, b)_{\infty}$ with Jacobi symbol $(-)_{J}$ and Hilbert symbol $(,)_{\infty}$ at the infinite place, and $\varepsilon_{d}$ 
is 1 or $i$ according as $d \equiv 1(\bmod 4)$ or $d \equiv-1(\bmod 4)$. Then

$\lambda_{1}(-a / c)=i \varepsilon_{a}\left(\frac{-2 c}{a}\right)$, if $c$ is a even positive integer and $(a, c)=1$,

$\lambda_{1}(-a / c)=0$, if $a$ and $c$ are odd, $c>0$ and $(a, c)=1$,

$\lambda_{1}(-a / c)=\sqrt{i} \varepsilon_{c}\left(\frac{2 a}{c}\right)$, if $c$ is a odd positive integer and $a$ is even.

Now let us define Eisenstein series by

$$
\begin{aligned}
& \varphi_{x}(z, s)=v^{s / 2} \sum_{\substack{(a, c)=1 \\
c>0}} \lambda_{1}(-a / c) \chi(c)(c z-a)^{-1 / 2}|c z-a|^{-s}, \\
& \psi_{x}(z, s)=v^{s / 2} \sum_{\substack{(a, c)=1 \\
c>0 ; o \text { odd }}} \lambda_{1}(-a / c) \chi(c)(c z-a)^{-1 / 2}|c z-a|^{-s}, \\
& \phi_{x}(z, s)=v^{s / 2} \sum_{\substack{(a, c)=1 \\
c>0 ; \text { even }}} \lambda_{1}(-a / c) \chi(c)(c z-a)^{-1 / 2}|c z-a|^{-s},
\end{aligned}
$$

where the sum in the first line is extended over all integers $a, c$ such that $(a, c)=1$ and $c>0$; the sum in the second line is extended over all integers $a, c$ such that $(a, c)=1, c$ is odd and $c>0$; and the sum in the last line is extended over all integers $a, c$ such that $(a, c)=1, c$ is even and $c>0$. Then, by Lemma 2 , we have obviously

$$
\varphi_{x}(z, s)=\psi_{x}(z, s)+\phi_{x}(z, s) .
$$

Put $\Theta(z)=\sum_{n=-\infty}^{\infty} e^{\pi i n^{2} z}, j^{\prime}(\gamma, z)=\Theta(\gamma z) / \Theta(z)$ and $J(\gamma, z)=c z+d$ for $\gamma=\left(\begin{array}{ll}a & b \\ c & d\end{array}\right) \in G$. Then it holds as well known that $j^{\prime}(\gamma, z)=\varepsilon_{d}^{-1}\left(\frac{2 c}{d}\right) J(\gamma, z)^{1 / 2}$ for $\gamma=\left(\begin{array}{ll}a & b \\ c & d\end{array}\right) \in \Gamma(2)$. Put

$$
E^{\chi p}(z, s)=\sum_{\gamma \in \Gamma(2) \infty \backslash \Gamma_{0}(2 p, 2)} j^{\prime}(\gamma, z)^{-1} \chi_{p}(d)|J(\gamma, z)|^{-s},
$$

where $\chi_{p}$ is a character defined by $\chi_{p}(n)=\left(\frac{n}{p}\right) \chi(n), \Gamma(2)_{\infty}=\left\{\left(\begin{array}{ll}a & b \\ c & d\end{array}\right) \in \Gamma(2)\right.$ $\mid c=0\}$ and $\Gamma_{0}(N, M)=\left\{\left(\begin{array}{ll}a & b \\ c & d\end{array}\right) \in \Gamma_{0}(N) \mid b \equiv 0(\bmod M)\right\} . \quad$ Then one can easily derive from Lemma 2 that

$$
\begin{gathered}
\psi_{x}(z, s)=v^{s / 2} \sqrt{i} \sqrt{z^{-1}}|z|^{-s} E^{\chi_{p}}(-1 / p z, s), \\
\phi_{x}(z, s)=v^{s / 2} p^{(1+2 s) / 2} \chi(2) j^{\prime}\left(\mu_{p}, p z\right)^{-1}\left|J\left(\mu_{p}, p z\right)\right|^{-s} E^{\chi p}\left(\mu_{p} p z, s\right),
\end{gathered}
$$

with $\mu_{p}=\left(\begin{array}{cc}p m & 2 n \\ 2 & p\end{array}\right)$ where $m, n$ are integers such that $p^{2} m-4 n=1$. 
Now let us compute the Fourier expansions of $\varphi_{x}(z, s), \psi_{x}(z, s)$ after [3]. Observing that $\lambda_{1}(a+2 c / c)=\lambda_{1}(a / c)$ for $a, c \in Z$ coprime to each other, we have

$$
\begin{aligned}
\varphi_{x}(2 z, s)= & (2 v)^{s / 2} \sum_{\substack{(a, c)=1 \\
c>0}} \lambda_{1}(a / c) \chi(c) c^{-s-1 / 2}(2 z+a / c)^{-(s+1) / 2}(2 \bar{z}+a / c)^{-s / 2} \\
= & (2 v)^{s / 2} \sum_{a / c \bmod 2} \sum_{n=-\infty}^{\infty} \lambda_{1}(a / c+2 n) \chi(c) c^{-s-1 / 2} \\
& \times(2 z+a / c+2 n)^{-(s+1) / 2}(2 \bar{z}+a / c+2 n)^{-s / 2} \\
= & (2 v)^{s / 2} \sum_{a / c \bmod 2} \lambda_{1}(a / c) \chi(c)(2 c)^{-s-1 / 2} \\
& \times \sum_{n=-\infty}^{\infty}(z+a / 2 c+n)^{-(s+1) / 2}(\bar{z}+a / 2 c+n)^{-s / 2}
\end{aligned}
$$

Since

$$
\sum_{n=-\infty}^{\infty}(z+n)^{-(s+1) / 2}(\bar{z}+n)^{-s / 2}=\sum_{n=-\infty}^{\infty} i^{-1 / 2} v^{-s+1 / 2} \rho_{n}(s, v) e^{2 \pi i n u}
$$

with

$$
\rho_{n}(s, v)=\int_{-\infty}^{\infty} \frac{e^{-2 \pi i n v \xi} d \xi}{(1-i \xi)^{(s+1) / 2}(1+i \xi)^{s / 2}},
$$

we have

$$
\begin{aligned}
\varphi_{x}(z, s) & =2^{s / 2} v^{-s / 2+1 / 2} \sum_{\substack{a / \bmod 2 \\
c>0}} \lambda_{1}(a / c) \chi(c)(2 c)^{-1 / 2-s} \sum_{n=-\infty}^{\infty} i^{-1 / 2} \rho_{n}(s, v) e^{2 \pi i n(u+a / 2 c)} \\
& =2^{s / 2} v^{-(s-1) / 2} \sum_{n=-\infty}^{\infty} j_{1}(n, s) \rho_{n}(s, v) e^{2 \pi i n u}
\end{aligned}
$$

where

$$
\begin{aligned}
j_{1}(n, s) & =\sum_{\substack{a / c \bmod 2 \\
c>0}} \lambda_{1}(a / c) \chi(c)(2 c)^{-1 / 2-s} i^{-1 / 2} e^{\pi i n a / c} \\
& =2^{-s-3 / 2} \sum_{c=1}^{\infty} c^{-s-1} \chi(c) \sum_{h=1}^{2 c} \sum_{\substack{\text { modd } 2 c \\
(a, c)=1}} e^{-\pi i\left(h^{2}-n ; a / c\right.} .
\end{aligned}
$$

Put $L\left(s,{ }_{-} \chi\right)=\sum_{n=1}^{\infty} \chi(n) n^{-s}$, then

$$
L(s, \chi) \dot{j}_{1}(n, s)=2^{-s-1 / 2} \sum_{b=1}^{\infty} b^{-s} \chi(b) A_{2 b}(n) .
$$

Put

$$
\xi_{p}(s, n)=\sum_{r=0}^{\infty} p^{-r s} \chi\left(p^{r}\right) A_{p^{r}}(n),
$$


for all primes $p$ and put

$$
\eta_{2}(s, n)=\sum_{r=0}^{\infty} 2^{-r s} \chi\left(2^{r}\right) A_{2^{r+1}}(n),
$$

then $\xi_{2}(s, n)=1+2^{-s} \chi(2) \eta_{2}(s, n)$ and

$$
L(s, \chi) \boldsymbol{j}_{1}(n, s)=2^{-s-1 / 2}\left(\prod_{p \neq 2} \xi_{p}(s, n)\right) \eta_{2}(s, n) .
$$

In the exactly same way we have

$$
\psi_{x}(z, s)=2^{s / 2} v^{-(s-1) / 2} \sum_{n=-\infty}^{\infty} \boldsymbol{j}(n, s) \rho_{n}(s, v) e^{2 \pi i n u},
$$

where

$$
j(n, s)=\sum_{\substack{a / c \bmod 2 \\ c>0 ; \text { odd }}} \lambda_{1}(a / c) \chi(c)(2 c)^{-1 / 2-s} i^{-1 / 2} e^{\pi i n a / c} .
$$

Put $L_{2}(s, \chi)=\sum_{\substack{n=1 \\ n ; \text { odd }}}^{\infty} \chi(n) n^{-s}$, then

$$
L_{2}(s, \chi) \boldsymbol{j}(n, s)=2^{-s-1 / 2} \sum_{\substack{b=1 \\ b ; \text { odd }}}^{\infty} b^{-s} \chi(b) A_{2 b}(n)=2^{-s-1 / 2} \prod_{p \neq 2} \xi_{p}(s, n) .
$$

Since $E(s, n)=\prod_{p} \xi_{p}(s, n)$ and $\xi_{2}(s, n)=1+2^{-s} \chi(2) \eta_{2}(s, n)$,

$$
\begin{aligned}
L_{2}(s, \chi) \psi_{\chi}(2 z, s)+2^{-s} \chi(2) L(s, \chi) \varphi_{\chi}(2 z, s) \\
=2^{-s / 2-1 / 2} v^{-(s-1) / 2} \sum_{n=-\infty}^{\infty} e^{2 \pi i n u} \rho_{n}(s, v) E(s, n) .
\end{aligned}
$$

Recalling that

$$
E_{\theta}(z, s)=2^{s / 2} \Gamma((s+1) / 2)(2 \pi v)^{-(s+1) / 2} \sqrt{v} \sum_{n \in \boldsymbol{Z}} e^{2 \pi i n u} \rho_{n}(s+1, v) \Xi(s+1, n),
$$

we obtain

Theorem 1. The integral $E_{\theta}(z, s)$, defined in (1.29), of the theta function for the ternary quadratic form defined in (1.26) is a linear combination of Eisenstein series $\varphi_{x}$ and $\psi_{x}$ defined in (2.2) and (2.3), that is,

$$
\begin{aligned}
& E_{\theta}(z, s)=2^{s+1} \Gamma((s+1) / 2)(2 \pi)^{-(s+1) / 2} \\
& \quad \times\left(L_{2}(s, \chi) \psi_{\chi}(2 z, s)+2^{-s} \chi(2) L(s, \chi) \varphi_{\chi}(2 z, s)\right) .
\end{aligned}
$$

3. We continue to compute the right-hand side of the equality (1.22) in this section. First, by changing the variable $z$ to $-1 / p z$ and using (1.23), (1.28), (1.29), (1.30), we can transform it to the following forms: 


$$
\begin{aligned}
\sqrt{p}^{-1} & \int_{\Gamma \backslash H} \varphi(-1 / p z) \int_{0}^{\infty} \int_{0}^{1} \tilde{\theta}(z, w, w) y^{s-1} d x d y d_{0} z \\
= & \sqrt{p}^{-1} \int_{\Gamma \backslash H} \varphi(-1 / p z) E_{I}(z, s) d_{0} z \\
= & \sqrt{p}^{-1} \int_{\Gamma \backslash H}\left(\sqrt{v} \varphi(-1 / p z) \overline{\theta(z)} E_{\theta}(z, s)\right. \\
& \left.+\sqrt{v} \varphi(-1 / p z) \overline{\theta_{0}(z)} E_{\theta, 0}(z, s)\right) d_{0} z .
\end{aligned}
$$

Note that $\theta(z)$ and $E_{\theta}(z, s)$ are automorphic under $\Gamma_{0}(4 p)$; and so is $\overline{\theta_{0}(z)} E_{\theta, 0}(z, s)$ because

$$
\tilde{\Theta}(z, g, g)=v^{1 / 2} \overline{\theta(z)} \Theta_{3}(z, g)+v^{1 / 2} \overline{\theta_{0}(z)} \Theta_{3,0}(z, g)
$$

is automorphic under $\Gamma_{0}(p)$. Therefore we can decompose the integral in (3.1) as follows.

$$
\begin{gathered}
\sqrt{p}^{-1}\left[\Gamma_{0}(p): \Gamma_{0}(4 p)\right]^{-1}\left(\int_{\mathscr{D}} \sqrt{v} \varphi(-1 / p z) \overline{\theta(z)} E_{\theta}(z, s) d_{0} z\right. \\
\left.\quad+\int_{\mathscr{D}} \sqrt{v} \varphi(-1 / p z) \overline{\theta_{0}(z)} E_{\theta, 0}(z, s) d_{0} z\right)
\end{gathered}
$$

where $\mathscr{D}$ denotes the fundamental domain $\Gamma_{0}(4 p) \backslash H$. To investigate the second integral, consider

$$
\Theta_{3,0}(z, g)=\sqrt{v} \sum_{X=\left(\begin{array}{ll}
a & b \\
b & c
\end{array}\right) \in L_{3,0}} \chi(a) e^{2 \pi i(-u \operatorname{det} X+i 2-1 v \operatorname{tr}(\langle t g X g) 2))},
$$

then we see that

$$
\Theta_{3,0}(z, g)=\Theta_{3}^{*}(z, g)+\Theta_{3}(z, g)
$$

when we put

$$
\Theta_{3}^{*}(z, g)=\sqrt{v} \sum_{X=\left(\begin{array}{c}
a b \\
b c
\end{array}\right) \in L_{3}^{*}} \chi(a) e^{2 \pi i\left(-u \operatorname{det} X+i^{2}-1 v \operatorname{tr}\left(\langle t g X g)^{2}\right)\right)},
$$

with $L_{3}^{*}=\left\{\left(\begin{array}{cc}a & b / 2 \\ b / 2 & c\end{array}\right) \mid a, b, c \in Z\right\}$. By [7],

$$
\Theta_{3}(\sigma z, g)=\sqrt{2 i^{-1}} \sqrt{p z+4} \chi(4) \Theta_{3}^{*}(z, g)
$$

with $\sigma=\left(\begin{array}{ll}4 m & n \\ p & 4\end{array}\right)$ where $m, n$ are integers such that $16 m-p n=1$. Since $\sigma$ normalizes $\Gamma_{0}(4 p, 4), \Theta_{3}^{*}(z, g)$ is automorphic with respect to $\Gamma_{0}(4 p, 4)$. Namely, it holds $\Theta_{3}^{*}(\gamma z, g)=\chi(d) \sqrt{c z+d} \Theta_{3}^{*}(z, g)$ for $\gamma=\left(\begin{array}{ll}a & b \\ c & d\end{array}\right) \in \Gamma_{0}(4 p, 4)$. Hence we can decompose the second integral in (3.3) into two terms, that is, 


$$
\begin{aligned}
\int_{\mathscr{D}} \sqrt{v} \varphi & (-1 / p z) \overline{\theta_{0}(z)} E_{\theta, 0}(z, s) d_{0} z \\
= & \int_{\mathscr{D}} \sqrt{v} \varphi(-1 / p z) \overline{\theta_{0}(z)} \int_{0}^{\infty} \int_{0}^{1} \Theta_{3,0}\left(z,\left(\begin{array}{cc}
1 & x \\
0 & 1
\end{array}\right)\left(\begin{array}{cc}
\sqrt{y} & 0 \\
0 & 1 / \sqrt{y}
\end{array}\right)\right) d x y^{s-1} d y \\
= & 1 / 4 \int_{\mathscr{Q}^{\prime}} \sqrt{v} \varphi(-1 / p z) \overline{\theta_{0}(z)} \\
& \times \int_{0}^{\infty} \int_{0}^{1} \Theta_{3}^{*}\left(z,\left(\begin{array}{ll}
1 & x \\
0 & 1
\end{array}\right)\left(\begin{array}{cc}
\sqrt{y} & 0 \\
0 & 1 / \sqrt{y}
\end{array}\right)\right) d x y^{s-1} d y d_{0} z \\
& +1 / 4 \int_{\mathscr{Q}^{\prime}} \sqrt{v} \varphi(-1 / p z) \overline{\theta_{0}(z)} \\
& \times \int_{0}^{\infty} \int_{0}^{1} \Theta_{3}\left(z,\left(\begin{array}{ll}
1 & x \\
0 & 1
\end{array}\right)\left(\begin{array}{cc}
\sqrt{y} & 0 \\
0 & 1 / \sqrt{y}
\end{array}\right)\right) d x y^{s-1} d y d_{0} z
\end{aligned}
$$

with $\mathscr{D}^{\prime}=\Gamma_{0}(4 p, 4) \backslash H . \quad$ By (3.7) the first term is equal to

$$
1 / 4 \int_{\mathscr{Q}^{\prime}} \sqrt{v} \varphi(-1 / p z) \overline{\theta_{0}(z)} \sqrt{2} \bar{i}(p z+4)^{-1 / 2} \bar{\chi}(4) E_{\theta}(\sigma z, s) d_{0} z
$$

and since $\sigma \mathscr{D}^{\prime}$ is also a fundamental domain of $\Gamma_{0}(4 p)$ and can be taken as the domain of integration in (3.9) instead of $\mathscr{D}^{\prime}$, it is transformed to

$$
1 / 4 \int_{\mathscr{Q}^{\prime}} \sqrt{v}|p z-4 m|^{-1} \varphi\left(\omega_{p} \sigma^{-1} z\right) \overline{\theta_{0}\left(\sigma^{-1} z\right)} \sqrt{2 i}\left(p \sigma^{-1} z+4\right)^{-1 / 2} \bar{\chi}(4) E_{\theta}(z, s) d_{0} z
$$

with $\omega_{p}=\left(\begin{array}{rr}0 & -1 \\ p & 0\end{array}\right) . \quad$ Put

$$
\Theta_{1}(z)=\sum_{n=-\infty}^{\infty} e^{\pi i n 2 z}, \quad \Theta_{2}(z)=\sum_{n=-\infty}^{\infty}(-1)^{n} e^{\pi i n^{2} z}, \quad \Theta_{3}(z)=\sum_{n=-\infty}^{\infty} e^{\pi i(n+1 / 2)^{2 z}},
$$

then the well known transformation formulas

$$
\begin{array}{ll}
\Theta_{1}(z+1)=\Theta_{2}(z), & \Theta_{2}(z+1)=\Theta_{1}(z), \\
\Theta_{3}(z+1)=\sqrt{i} \Theta_{3}(z), & \Theta_{1}(-1 / z)=\sqrt{z / i} \Theta_{1}(z), \\
\Theta_{2}(-1 / z)=\sqrt{z / i} \Theta_{3}(z), & \Theta_{3}(-1 / z)=\sqrt{z / i} \Theta_{3}(z),
\end{array}
$$

hold. Using these and the explicit transformation formula for $\Theta_{1}(z)=$ $\theta\left(2^{-1} z\right)$,

$$
\Theta_{1}(\gamma z)=\left(\frac{2 c}{d}\right) \varepsilon_{d}^{-1} \sqrt{c z+d} \Theta_{1}(z) \text { for } \gamma=\left(\begin{array}{ll}
a & b \\
c & d
\end{array}\right) \in \Gamma(2)
$$

we have 


$$
\begin{aligned}
& \theta_{0}\left(\sigma^{-1} z\right)=\Theta_{3}\left(\alpha \sigma^{-1} z\right)=\Theta_{3}\left(\omega^{-1} \sigma^{\prime} \alpha^{-1} z\right) \\
& =i^{-1 / 2}\left(\sigma^{\prime} \alpha^{-1} z\right)^{1 / 2} \Theta_{2}\left(\sigma^{\prime} \alpha^{-1} z\right)=i^{-1 / 2}\left(\sigma^{\prime} \alpha^{-1} z\right)^{1 / 2} \Theta_{1}\left(\tilde{\sigma} \varepsilon \alpha^{-1} z\right) \\
& =i^{-1 / 2}\left(\sigma^{\prime} \alpha^{-1} z\right)^{1 / 2} \varepsilon_{-n-8}^{-1}\left(8 \varepsilon \alpha^{-1} z-n-8\right)^{1 / 2} \Theta_{1}\left(\varepsilon \alpha^{-1} z\right) \\
& =\sqrt{2 i^{-1}} \sqrt{p z-4 m} \Theta_{2}\left(2^{-1} z\right)
\end{aligned}
$$

where $\sigma^{\prime}=\omega \alpha \sigma^{-1} \alpha\left(\begin{array}{cc}1 / 2 & 0 \\ 0 & 1 / 2\end{array}\right)=\left(\begin{array}{cc}p & -2 m \\ 8 & -n\end{array}\right), \quad \varepsilon=\left(\begin{array}{ll}1 & 1 \\ 0 & 1\end{array}\right), \quad \alpha=\left(\begin{array}{ll}2 & 0 \\ 0 & 1\end{array}\right)$ and $\tilde{\sigma}=$ $\varepsilon \sigma^{\prime} \varepsilon^{-1}=\left(\begin{array}{c}p+8 \\ 8-n-8\end{array}\right)$. Here notice that $n \equiv-1(\bmod 4)$ in $(3.14)$ since $16 m-p n=1$; therefore $\varepsilon_{-n-8}=1$. Observing that $\varphi\left(\omega_{p} \sigma^{-1} z\right)=\chi(4) \varphi\left(\omega_{p} z\right)$, we see that the first term of (3.8) equals

$$
1 / 4 \int_{\mathscr{Q}^{\prime}} \sqrt{v} \varphi\left(\omega_{p} z\right) \overline{\Theta_{2}\left(2^{-1} z\right)} E_{\theta}(z, s) d_{0} z
$$

Put $\gamma_{i}=\left(\begin{array}{ll}1 & i \\ 0 & 1\end{array}\right),(i=1,2,3,4)$, then $\Gamma_{0}(4 p)=\bigcup_{i=1}^{4} \Gamma_{0}(4 p, 4) \gamma_{i}$. Hence we can suppose that $\mathscr{D}^{\prime}=\cup_{i=1}^{4} \gamma_{i} \mathscr{D}$. Therefore (3.15) becomes

$$
1 / 4 \int_{\mathscr{2}} \sqrt{v} \varphi\left(\omega_{p} z\right) \sum_{i=1}^{4} \overline{\Theta_{2}\left(\left(\begin{array}{ll}
1 & i \\
0 & 2
\end{array}\right) z\right)} E_{\theta}(z, s) d_{0} z
$$

By the definition of $\Theta_{2}(z)$, we have $\sum_{i=1}^{4} \overline{\Theta_{2}\left(\left(\begin{array}{ll}1 & i \\ 0 & 2\end{array}\right) z\right)}=4 \theta(z)$ and therefore (3.16) becomes

$$
\int_{\mathscr{D}} \sqrt{v} \varphi\left(\omega_{\eta} z\right) \theta(z) E_{\theta}(z, s) d_{0} z
$$

which is identical with the first integral in (3.3).

We show that the second term of (3.8)

$$
1 / 4 \int_{\mathscr{Q}^{\prime}} \sqrt{v} \varphi(-1 / p z) \overline{\theta_{0}(z)} E_{\theta}(z, s) d_{0} z
$$

is 0 . Since $\mathscr{D}^{\prime}=\cup_{i=1}^{4} \gamma_{i} \mathscr{D}$ and $\theta_{0}(z)=\Theta_{3}(2 z)$, it equals

$$
1 / 4 \int_{\mathscr{D}} \sqrt{v} \varphi\left(\omega_{p} z\right) \sum_{i=1}^{4} \overline{\Theta_{3}\left(\left(\begin{array}{cc}
2 & 2 i \\
0 & 1
\end{array}\right) z\right)} E_{\theta}(z, s) d_{0} z
$$

which is 0 because $\sum_{i=1}^{4} \overline{\Theta_{3}\left(\left(\begin{array}{cc}2 & 2 i \\ 0 & 1\end{array}\right) z\right)}=0$ by the definition (3.11). Thus we obtain

$$
\begin{aligned}
& \int_{0}^{\infty} \int_{0}^{1} F(w, w) d x y^{s-1} d y \\
& \quad=2 p^{-1} g(\bar{\chi})\left[\Gamma_{0}(p): \Gamma_{0}(4 p)\right]^{-1} \int_{\mathscr{D}} \sqrt{v} \varphi(-1 / p z) \overline{\theta(z)} E_{\theta}(z, s) d_{0} z
\end{aligned}
$$


By Theorem 1 and (2.5), we have

$$
\begin{aligned}
& E_{\theta}(z, s)=\Gamma((s+1) / 2)(2 \pi)^{-(s+1) / 2} \chi(2) L(s, \gamma) \\
& \quad \times\left(\bar{\chi}(2) 2^{s+1} \psi_{\chi}(2 z, s+1)+\phi_{\chi}(2 z, s+1)\right) .
\end{aligned}
$$

Therefore by (2.7) and (2.8) we have

$$
\begin{aligned}
\int_{\mathscr{L}} \sqrt{v} & \varphi(-1 / p z) \overline{\theta(z)} E_{\theta}(z, s) d_{0} z \\
= & \Gamma((s+1) / 2)(2 \pi)^{-(s+1) / 2} \chi(2) L(s+1, \chi) \\
& \times\left(2^{(s+1) / 2} p^{s+3 / 2} \chi(2) P_{1}(s)+\bar{\chi}(2) 2^{3(s+1) / 2} \sqrt{i} P_{2}(s)\right)
\end{aligned}
$$

where

$$
\begin{aligned}
& P_{1}(s)=\int_{\mathscr{Q}} \varphi(-1 / p z) \overline{\theta(z)} v^{s / 2+1} j^{\prime}\left(\mu_{p}, 2 p z\right)^{-1} \\
& \times\left|J\left(\mu_{p}, 2 p z\right)\right|^{-s-1} E^{x_{p}}\left(\mu_{p} 2 p z, s+1\right) d_{0} z, \\
& P_{2}(s)=\int_{\mathscr{D}} \varphi(-1 / p z) \overline{\theta(z)} v^{s / 2+1} \\
& \times \sqrt{2 z^{-1}}|2 z|^{-s-1} E^{\chi p}(-1 / 2 p z, s+1) d_{0} z .
\end{aligned}
$$

Put $\nu=\left(\begin{array}{cc}p & -n \\ -4 p & p^{2} m\end{array}\right)$, then $\nu$ normalizes $\Gamma_{0}(4 p)$ and we can take $\nu^{-1} \mathscr{D}$ as a domain of integration instead of $\mathscr{D}$. Hence, changing the variable $z$ to $\nu z$, we have

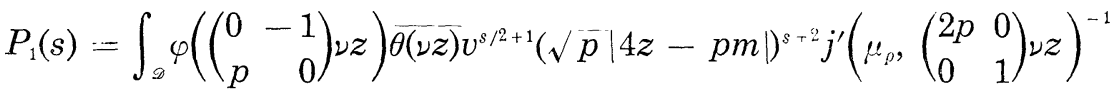

$$
\begin{aligned}
& \times\left|J\left(\mu_{p},\left(\begin{array}{cc}
2 p & 0 \\
0 & 1
\end{array}\right) \nu z\right)\right|^{-s-1} E^{\chi_{p}}\left(\mu_{p}\left(\begin{array}{cc}
2 p & 0 \\
0 & 1
\end{array}\right) \nu z, s+1\right) d_{0} z .
\end{aligned}
$$

Since $\mu_{p}\left(\begin{array}{ll}2 p & 0 \\ 0 & 1\end{array}\right) \nu=\left(\begin{array}{ll}2 & 0 \\ 0 & 1\end{array}\right),\left(\begin{array}{rr}0 & -1 \\ p & 0\end{array}\right) \nu\left(\begin{array}{ll}p & 0 \\ 0 & p\end{array}\right)^{-1}=\left(\begin{array}{lr}4 & -p m \\ p & -n\end{array}\right) \in \Gamma_{0}(p)$ and $\nu\left(\begin{array}{ll}p & 0 \\ 0 & 1\end{array}\right)^{-1}$ $=\left(\begin{array}{rr}1 & -n \\ -4 & p^{2} m\end{array}\right) \in \Gamma_{0}(4)$, we have $E^{x_{p}}\left(\mu_{p}\left(\begin{array}{cc}2 p & 0 \\ 0 & 1\end{array}\right) \nu z, s+1\right)=E^{\gamma_{p}}(2 z, s+1)$, $\varphi\left(\left(\begin{array}{rr}0 & -1 \\ p & 0\end{array}\right) \nu z\right)=\chi(-n) \varphi(z)=\bar{\chi}(4) \varphi(z) \quad$ and $\quad \theta(\nu z)=\left(-4 p z+p^{2} m\right)^{1 / 2} \theta(p z)$.

Therefore we obtain

$$
P_{1}(s)=p^{-(s+1) / 2} \bar{\chi}(4) \int_{\mathscr{Q}} \varphi(z) \overline{\theta(p z)} v^{s / 2+1} E^{\chi_{p}}(2 z, s+1) d_{v} z .
$$

As for $P_{2}(s)$ in (3.24), changing the variable $z$ to $-1 / 4 p z$, we have

$$
P_{2}(s)=p^{(s+2) / 2} \sqrt{i^{-1}} \int_{\mathscr{D}} \varphi(4 z) \overline{\theta(p z)} v^{s / 2+1} E^{x p}(2 z, s+1) d_{1,} z .
$$


Hence it follows from (2.22) that

$$
\begin{aligned}
\int_{\mathscr{\mathscr { \omega }}} \sqrt{ } \bar{v} \varphi & (-1 / p z) \overline{\theta(z)} E_{\theta}(z, s) d_{0} z \\
= & \Gamma((s+1) / 2)(2 \pi)^{-(s+1) / 2} L(s+1, \chi) p^{(s+2) / 2} \\
& \times\left(2^{(s+1) / 2} \int_{\mathscr{D}} \varphi(z) \overline{\theta(p z)} v^{s / 2+1} E^{\chi_{\boldsymbol{p}}^{\prime}}(2 z, s+1) d_{0} z\right. \\
& \left.+2^{3(s+1) / 2} \int_{\mathscr{D}} \varphi(4 z) \overline{\theta(p z)} v^{s / 2+1} E^{x_{\boldsymbol{p}}^{\prime}}(2 z, s+1) d_{0} z\right) .
\end{aligned}
$$

Denote the first (respectively the second) integral in (3.28) by $Q_{1}(s)$ (respectively $Q_{2}(s)$ ). Then the usual computation for convolutions gives that for $\varphi(z)=\sum_{n \neq 0} a_{|n|} v^{1 / 2} K_{\nu}(2 \pi|n| v) e^{2 \pi n i u}$,

$$
\begin{aligned}
Q_{1}(s) & =\int_{\mathscr{D}} \varphi(z) \overline{\theta(p z)} v^{s / 2+1} E^{x_{p}}(2 z, s+1) d_{0} z \\
& =\sum_{r \in\left\langle\Gamma_{\infty} \backslash \Gamma_{0}\right)(4 p)} \int_{\mathscr{D}} v\langle\gamma z\rangle^{(s+2) / 2} \varphi(\gamma z) \overline{\theta(p \gamma z)} d_{0} z \\
& =\int_{0}^{\infty} \int_{0}^{1} v^{(s+2) / 2} \varphi(z) \overline{\theta(p z)} d_{0} z \\
& =\int_{0}^{\infty} 2 \sum_{n=1}^{\infty} a_{p n 2} K_{\nu}\left(2 \pi p n^{2} v\right) e^{-2 \pi p n^{2} v} v^{s / 2-1 / 2} d v \\
& =2(2 \pi)^{-(s+1) / 2} \sum_{n=1}^{\infty} a_{p n^{2}}\left(p n^{2}\right)^{-(s+1) / 2} \int_{0}^{\infty} v^{(s-1) / 2} K_{\nu}(v) e^{-v} d v
\end{aligned}
$$

where $\Gamma_{\infty}=\left\{ \pm\left(\begin{array}{ll}1 & n \\ 0 & 1\end{array}\right) \mid n \in Z\right\}$. By the same reasoning,

$$
\begin{aligned}
Q_{1}(s) & =\int_{0}^{\infty} \int_{0}^{1} v^{(s+2) / 2} \varphi(4 z) \overline{\theta(p z)} d_{0} z \\
& =\int_{0}^{\infty} 4 \sum_{n=1}^{\infty} a_{p n^{2}} K_{\nu}\left(8 \pi p n^{2} v\right) e^{-8 \pi p n^{2} v} v^{s / 2-1 / 2} d v \\
& =4(8 \pi)^{-(s+1) / 2} \sum_{n=1}^{\infty} a_{p n^{2}}\left(p n^{2}\right)^{-(s+1) / 2} \int_{0}^{\infty} v^{(s-1) / 2} K_{\nu}(v) e^{-v} d v,
\end{aligned}
$$

hence we have

$$
\begin{aligned}
\int_{0} \sqrt{v} \varphi( & -1 / p z) \overline{\theta(z)} E_{\theta}(z, s) d_{0} z \\
= & \Gamma((s+1) / 2)(2 \pi)^{-(s+1) / 2} L(s+1, \chi) p^{(s+2) / 2} \\
& \times 2(2 \pi)^{-(s+1) / 2} \sum_{n=1}^{\infty} a_{p n^{2}}\left(p n^{2}\right)^{-(s+1) / 2} \int_{0}^{\infty} v^{(s-1) / 2} K_{\nu}(v) e^{-v} d v \\
& \times\left(2^{(s+1) / 2}+2 \cdot 4^{-(s+1) / 2} 2^{3(s+1) / 2}\right) \\
= & 6 \Gamma((s+1) / 2)(2 \pi)^{-(s+1)} L(s+1, \chi) p^{1 / 2} a_{p} 2^{(s+1) / 2}
\end{aligned}
$$




$$
\times\left(\sum_{n=1}^{\infty} a_{n^{2}} n^{-(s+1)}\right) \int_{0}^{\infty} v^{(s-1) / 2} K_{\nu}(v) e^{-v} d v .
$$

Therefore we finally obtain

$$
\begin{aligned}
& \int_{0}^{\infty} \int_{0}^{1} F(w, w) d x y^{s-1} d y \\
&= \Gamma((s+1) / 2)(2 \pi)^{-(s+1)} L(s+1, \chi) a_{p} 2^{(s+3) / 2} p^{-1 / 2} g(\bar{\chi}) \\
& \times\left(\sum_{n=1}^{\infty} a_{n^{2}} n^{-(s+1)}\right) \int_{0}^{\infty} v^{(s-1) / 2} K_{\nu}(v) e^{-v} d v
\end{aligned}
$$

Comparing this with (1.21), we have

$$
\begin{aligned}
& c \int_{0}^{\infty} K_{\nu}(y)^{2} y^{s-1} d y \\
& \quad=\Gamma((s+1) / 2) a_{p} 2^{(s+3) / 2} p^{-1 / 2} g(\bar{\chi}) \int_{0}^{\infty} v^{(s-1) / 2} K_{\nu}(v) e^{-v} d v .
\end{aligned}
$$

From the well known formulas (see [5], p. 101 and p. 37)

$$
\begin{aligned}
& \text { (3.34) } \begin{aligned}
\int_{0}^{\infty} v^{(s-1) / 2} K_{\nu}(v) e^{-v} d v \\
\quad=\sqrt{\pi} 2^{-(s+1) / 2} \Gamma(s / 2+1 / 2+\nu) \Gamma(s / 2+1 / 2-\nu) \Gamma(s / 2+1)^{-1}
\end{aligned} \\
& \text { (3.35) } \begin{aligned}
\int_{0}^{\infty} K_{\nu}(y)^{2} y^{s-1} d y \\
=
\end{aligned} \\
& =2^{s-2} \Gamma(s / 2+1 / 2+\nu) \Gamma(s / 2+1 / 2-\nu) \Gamma(s / 2+1 / 2)^{2} \Gamma(s+1)^{-1}
\end{aligned}
$$

and $\Gamma(2 s)=2^{2 s-1} \pi^{-1 / 2} \Gamma(s) \Gamma(s+1 / 2)$,

$$
c=2^{3} a_{p} p^{-1 / 2} g(\bar{\chi})
$$

follows.

We can easily verify the following lemma:

LEMMA 3. Let $\varphi(z)=\sum_{n \neq 0} a_{|n|} y^{1 / 2} K_{\nu}(2 \pi|n| y) e^{2 \pi n \imath x}$ be a cusp form with the character $\chi$, which is a common eigenfunction for all Hecke operators, and assume $a_{1}=1$. Define the cusp form $\phi$ with the character $\bar{\chi}$ by $\phi(z)$ $=\varphi(-1 / p z)$. Then $\phi$ is a common eigenfunction for all Hecke operators and its first Fourier coefficient $\lambda_{\varphi}$ is given by

$$
\lambda_{\varphi}==\mathfrak{g}(\bar{\chi}) a_{p}^{-1} .
$$

Let $\phi$ be as in Lemma 3 and put

$$
G\left(w_{1}, w_{2}\right)=\int_{\Gamma \backslash H} \phi(z) \tilde{\theta}\left(z, w_{1}, w_{2}\right) d_{0} z
$$


with $\Gamma=\Gamma_{0}(p)$. Then by (1.3), (1.17), (1.19) and (3.36) we have

$$
G\left(w_{1}, w_{2}\right)=\mathfrak{g}(\bar{\chi}) F\left(w_{1}, w_{2}\right)=2^{3} a_{p} \sqrt{p} \varphi\left(w_{1}\right) \varphi\left(w_{2}\right) .
$$

Put $\psi(z)=\lambda_{\varphi}^{-1} \phi(z)$, then $\psi$ is a common eigenfunction for all Hecke operators whose first Fourier coefficient is unity and therefore

$$
\int_{\Gamma \backslash H} \psi(z) \tilde{\theta}\left(z,-1 / p w_{1},-1 / p w_{2}\right) d_{0} z=\lambda_{\varphi}^{-1} 2^{3} a_{p} \sqrt{p} \varphi\left(-1 / p w_{1}\right) \varphi\left(-1 / p w_{2}\right) .
$$

Hence we obtain

TheOREm 2. Let $\psi$ be a cusp form with the character $\bar{\chi}$, which is a common eigenfunction for all Hecke operators. Assume that the first Fourier coefficient of $\psi$ is unity. Then it holds

$$
\int_{\Gamma \backslash I I} \psi(z) \tilde{\theta}\left(z,-1 / p w_{1},-1 / p w_{2}\right) d_{0} z=2^{3} \sqrt{p} g(\bar{\chi}) \psi\left(w_{1}\right) \psi\left(w_{2}\right) .
$$

4. Let $\mathfrak{o}$ be the ring of integers in an imaginary quadratic field $\boldsymbol{Q}(\sqrt{-p})$ with a rational prime $p \equiv 1(\bmod 4)$. Then $\mathfrak{o}=Z+\sqrt{-p} Z$. Put $\mathfrak{P}=\sqrt{-p} \mathfrak{0}$ and denote the upper half space $\{(z, v) \mid z \in C, v>0\}$ by $H$. Let us define the action of $G_{C}=S L(2, C)$ on $H$ in the usual way. For $a \in C$, put $\tilde{a}=\left(\begin{array}{ll}a & 0 \\ 0 & \bar{a}\end{array}\right)$. For $w=(z, v) \in \boldsymbol{H}$ and $\gamma=\left(\begin{array}{ll}a & b \\ c & d\end{array}\right) \in G_{C}$, put $\tilde{w}$ $\left(\begin{array}{rr}z & -v \\ v & \bar{z}\end{array}\right)$, and define $w^{\prime}=\gamma w=\left(z^{\prime}, v^{\prime}\right) \in \boldsymbol{H}$ by the relation

$$
\tilde{w}^{\prime}=(\tilde{a} \tilde{w}+\tilde{b})(\tilde{c} \tilde{w}+\tilde{d})^{-1}
$$

Put

$$
E_{0}(w, s)=\sum_{n \in 0, m \in \Re} v^{s}\left(|m z+n|^{2}+|m|^{2} v^{2}\right)^{-s} \bar{\chi}(n)
$$

where $\chi$ is the character on $\mathfrak{o} / \mathfrak{P}$ defined by $\chi(n+\sqrt{-p} l)=\chi(n)$ for $n, l$ $\in Z$. Then $E_{0}$ is a non-holomorphic Eisenstein series on $\boldsymbol{H}$ satisfying $E_{\mathrm{o}}(\gamma w, s)=\chi(d) E_{\mathrm{o}}(w, s)$ for $\gamma=\left(\begin{array}{ll}a & b \\ c & d\end{array}\right) \in \Gamma_{\mathfrak{R}}=\left\{\left(\begin{array}{ll}a & b \\ c & d\end{array}\right) \in S L(2, \mathrm{o}) \mid c \equiv 0(\bmod \mathfrak{R})\right\}$.

Put

$$
F_{0}(w, s)=\sum_{n \in \Re, m \in 0} v^{s}\left(|m z+n|^{2}+|m|^{2} v^{2}\right)^{-s} \bar{\chi}(m)
$$

then $E_{0}(w, s)=F_{0}(\omega w, s)$ with $\omega=\left(\begin{array}{rr}0 & -1 \\ 1 & 0\end{array}\right) . \quad F_{0}$ has an usual integral expression, that is, 


$$
\begin{aligned}
& F_{\mathrm{o}}(w, s)=\pi^{s} \Gamma(s)^{-1} \\
& \quad \times \int_{0}^{\infty} \eta^{s-1} \sum_{n \in \mathfrak{*}, m \in \in_{0}} \bar{\chi}(m) \exp \left(-\pi \eta v^{-1}\left(|m z+n|^{2}+|m|^{2} v^{2}\right)\right) d \eta .
\end{aligned}
$$

The series in the integrand can be expressed as a theta function for a

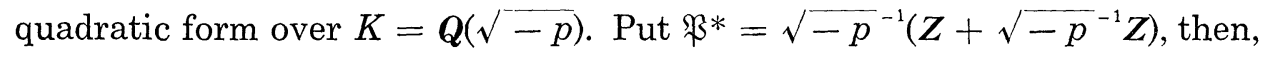
using the Poisson summation formula, we obtain

$$
\begin{aligned}
\sum_{n \in \mathfrak{B}} \exp \left(\pi \eta v^{-1}\left(|m z+n|^{2}+|m|^{2} v^{2}\right)\right) \\
=\sum_{n \in \mathfrak{P}^{*}} p^{-3 / 2} \eta^{-1} v \exp \left(-2 \pi i \Re e(m n z)-\pi\left(|m|^{2} \eta+|n|^{2} \eta^{-1}\right) v\right)
\end{aligned}
$$

so that

$$
\begin{aligned}
\sum_{n \in \mathfrak{*}, m \in 0} & \bar{\chi}(m) \exp \left(-\pi \eta v^{-1}\left(|m z+n|^{2}+|m|^{2} v^{2}\right)\right) \\
= & p^{-3 / 2} \eta^{-1} v \sum_{m \in 0, n \in \mathfrak{R} *} \bar{\chi}(m) \\
& \quad \times \exp \left(-2 \pi i \Re e(m n z)-\pi\left(|m|^{2} \eta+|n|^{2} \eta^{-1}\right) v\right)
\end{aligned}
$$

Hence we obtain

$$
\begin{aligned}
& F_{0}(w, s)=\pi^{s} \Gamma(s)^{-1} p^{-3 / 2} \int_{0}^{\infty} \eta^{s-2} \\
& \quad \times v \sum_{m \in 0, n \in \Re *} \bar{\chi}(m) \exp \left(-2 \pi i \Re e(m n z)-\pi\left(|m|^{2} \eta+|n|^{2} \eta^{-1}\right) v\right) d \eta .
\end{aligned}
$$

Now we consider the pullbacks of $E_{0}, F_{0}$. It is easy to see that for $z=$ $u+i v \in H$ and $\gamma=\left(\begin{array}{ll}a & b \\ c & d\end{array}\right) \in S L(2, R), \gamma(u, v)=\left(u^{\prime}, v^{\prime}\right)$ holds denoting $\gamma z=$ $\frac{a z+b}{c z+d}=u^{\prime}+i v^{\prime}$. Since $E_{0}(\gamma w, s)=\chi(d) E_{0}(w, s)$ for $\gamma=\left(\begin{array}{ll}a & b \\ c & d\end{array}\right) \in \Gamma_{\Re}$ and $\Gamma_{\Re} \cap S L(2, R)=\Gamma_{0}(p)$, we see that for $\gamma=\left(\begin{array}{ll}a & b \\ c & d\end{array}\right) \in \Gamma_{0}(p), E(\gamma(u+i v))=$ $\chi(d) E(u+i v)$ holds and for $\sigma \in \Gamma^{0}(p), F(\sigma(u+i v))=\bar{\chi}(d) F(u+i v)$ holds when we put $E(u+i v)=E_{\mathrm{o}}((u, v), s), F(u+i v)=F_{\mathrm{o}}((u, v), s)$ with $u, v \in$ $\boldsymbol{R}$. If $z=u \in \boldsymbol{R}$, the series in the right-hand side of (4.7) can be viewed as a theta function for a quaternary quadratic form over $\boldsymbol{Q}$. In fact, put $n=(-p)^{-1 / 2}\left(-b-a(-p)^{-1 / 2}\right)$ and $m=d+(-p)^{1 / 2} c$ with $a, b, c, d \in Z$, then

$$
\begin{aligned}
\operatorname{Re}(m n)=\operatorname{det} & \left(\begin{array}{cc}
a & b \\
c & d / p
\end{array}\right), \\
|m|^{2} \eta+|n|^{2} \eta^{-1} & =\left(d^{2}+p c^{2}\right) \eta+p^{-1}\left(b^{2}+p^{-1} a^{2}\right) \eta^{-1} \\
& =\operatorname{tr}\left({ }^{t}\left(h_{\eta}\left(\begin{array}{cc}
a & b \\
c & d / p
\end{array}\right) l_{p}\right)\left(h_{\eta}\left(\begin{array}{cc}
a & b \\
c & d / p
\end{array}\right) l_{p}\right)\right),
\end{aligned}
$$

with 


$$
h_{\eta}=\left(\begin{array}{cc}
\left(\eta p^{3 / 2}\right)^{-1 / 2} & 0 \\
0 & \left(\eta p^{3 / 2}\right)^{1 / 2}
\end{array}\right), \quad l_{p}=\left(\begin{array}{cc}
p^{-1 / 4} & 0 \\
0 & p^{1 / 4}
\end{array}\right)
$$

Thus we obtain

$$
F_{0}((u, v), s)=\pi^{s} \Gamma(s)^{-1} p^{-1 / 2} \int_{0}^{\infty} \eta^{s-2} \Theta\left((u+i v) / p, h_{\eta}, l_{p}\right) d \eta
$$

where $\Theta(z, g, h)$ is the theta function defined in (1.1). It follows from (1.3) that

$$
F_{\mathrm{o}}(\omega(u, v), s)=\pi^{s} \Gamma(s)^{-1} p^{-3 / 2} g(\bar{\chi}) \int_{0}^{\infty} \eta^{s-2} \tilde{\Theta}\left(u+i v, h_{\eta}, l_{p}\right) d \eta .
$$

Since $\omega \tilde{h}_{\eta}{ }^{t} \omega_{p}=h_{\eta}$ and $\omega_{p} l_{p} \omega^{-1}=l_{p}$ with $\omega=\left(\begin{array}{cc}0 & -1 \\ 1 & 0\end{array}\right), \omega_{p}=\left(\begin{array}{cc}0 & -p^{-1 / 2} \\ p^{1 / 2} & 0\end{array}\right)$ and $\tilde{h}_{\eta}=\left(\begin{array}{cc}\eta^{1 / 2} p^{1 / 4} & 0 \\ 0 & \eta^{-1 / 2} p^{-1 / 4}\end{array}\right)$, it holds

$$
\tilde{\Theta}\left(z, h_{\eta}, l_{p}\right)=\tilde{\Theta}\left(z, \omega_{p} \tilde{h}_{\eta}, \omega_{p} l_{p}\right) .
$$

Therefore, for a cusp form $\psi$, putting $\mathscr{D}_{1}=\Gamma_{0}(p) \backslash H$ and $z=u+i v$, we have

$$
\begin{aligned}
& \int_{\mathscr{Q}_{1}} E_{0}((u, v), s) \psi(z) d_{0} z \\
& \quad=p^{-3 / 2} \mathrm{~g}(\bar{\chi}) \pi^{s} \Gamma(s)^{-1} \int_{0}^{\infty} \eta^{s-2} \int_{\mathscr{Q}_{1}} \tilde{\Theta}\left(z, \omega_{p} \tilde{h}_{\eta}, \omega_{p} l_{p}\right) \psi(z) d_{0} z d \eta .
\end{aligned}
$$

On the other hand, Theorem 2 asserts

$$
\int_{\mathscr{I}_{1}} \tilde{\Theta}\left(z, \omega_{p} \tilde{h}_{\eta}, \omega_{p} l_{p}\right) \psi(z) d_{0} z d \eta=2^{3} \sqrt{p} \mathfrak{g}(\chi) \psi\left(p^{1 / 2} \eta i\right) \psi\left(p^{-1 / 2} i\right)
$$

Thus we obtain

$$
\begin{aligned}
\int_{\mathscr{Q}_{1}} E_{0} & ((u, v), s) \psi(z) d_{0} z \\
& =\pi^{s} \Gamma(s)^{-1} 2^{3} \psi\left(p^{-1 / 2} i\right) \int_{0}^{\infty} \eta^{s-2} \psi\left(p^{1 / 2} \eta i\right) d \eta .
\end{aligned}
$$

Since $\int_{0}^{\infty} K_{\nu}(\eta) \eta^{s-1} d \eta=2^{s-2} \Gamma((s-\nu) / 2) \Gamma((s+\nu) / 2)$, we finally have

TheOREm 3. Let $\chi$ be a primitive Dirichlet character modulo a prime $p \equiv 1(\bmod 4) . \quad$ Assume $\chi(-1)=1 . \quad$ Let $\psi(z)=\sum_{n \neq 0} a_{|n|} v^{1 / 2} K_{\nu}(2 \pi|n| v) e^{2 \pi n i u}$ be a cusp form with the character $\bar{\chi}$, which is a common eigenfunction for all Hecke operators, and assume $a_{1}=1$. Let $E_{0}(w, s)$ be the Eisenstein series defined by (4.1) on the upper half space. Then it holds 


$$
\begin{aligned}
\int_{\Gamma_{0}(p) \backslash H} & E_{\mathrm{o}}((u, v), s) \psi(u+i v) v^{-2} d u d v \\
= & \pi^{1 / 2} \Gamma(s)^{-1} \Gamma\left(\frac{s-1 / 2-\nu}{2}\right) \Gamma\left(\frac{s-1 / 2+\nu}{2}\right) \\
& \times 2^{2} \psi\left(p^{-1 / 2} i\right) p^{-(s-1) / 2} L(s-1 / 2, \psi)
\end{aligned}
$$

where $L(s, \psi)=\sum_{n=1}^{\infty} a_{n} n^{-s}$.

\section{REFERENCES}

[1] G. Shimura, On the holomorphy of certain Dirichlet series, Proc. London Math. Soc., 31 (1975) , 79-98.

[ 2 ] - On modular forms of half integral weight, Ann. of Math., 97 (1973) , 440-481.

[3] C. L. Siegel, Die Funktionalgleichungen einiger Dirichletscher Reihen, Math. Z., 63 (1956), 363-373 (=Abh. III, 228-238).

[4] H. Maass, Über eine neue Art von nichtanalitishen automorphen Funktionen und die Bestimmung Dirichletscher Reihen durch Functionalgleichungen, Math. Ann., 121 (1949), 141-183.

[5] W. Magunus, F. Oberhettinger and R. P. Soni, Formulas and theorems for the special functions of mathematical phisics, Springer Verlag, 1966.

[6] H. Naganuma, On the coincidence of two Dirichlet series associated with cusp forms of Hecke's "Neben"-type and Hilbert modular forms over a real quadratic field, J. Math. Soc. Japan, 25 (1973), 547-555.

[ 7 ] T. Shintani, On construction of holomorphic cusp forms of half integral weight, Nagoya Math. J., 58 (1975), 83-126.

[ 8 ] H. Yoshida, Siegel's modular forms and the arithmetic of quadratic forms, Invent. Math., 60 (1980), 193-248.

[9] — - On an explicit construction of Siegel modular forms of genus 2, Proc. of Japan Acad. 1979

[10] - On Siegel modular forms obtained from theta series, J. Reine Angew. Math., 352 (1984), 184-219.

[11] A. Weil, Sur certain groupes d'opérateur unitaires. Acta Math., 111 (1964), 143-211.

[12] — Sur la formule de Siegel dans la théorie des groupes classique, ibid., 113 (1965), 1-87.

[13] P. B. Garrett, Pullbacks of Eisenstein series, in Automorphic forms in several variables (Proceedings of a Taniguchi symposium), Birkhauser, 1984.

[14] - Decomposition of Eisenstein series: Triple product $L$-functions, Preprint.

[15] J. L. Waldspurger, Sur les coefficients de Fourier des formes modulaires de poids semi-entiers, J. Math. Pures Appl., 60 (1981).

[16] S. Niwa, Modular forms of half integral weight and the integral of certain thetafunctions, Nagoya Math. J., 56 (1974), 147-161.

[17] S. Rallis and G. Schiffman, On a relation between $\tilde{S} L_{2}$ cusp forms and cusp forms on tube domains associated to orthogonal groups, Trans. of Amer. Math. Soc., 263 (1981), 1-58.

Nagoya City College of Child Education

Owariasahi, 488

Japan 